\title{
Type of vegetarian diet, obesity and diabetes in adult Indian population
}

\author{
Sutapa Agrawal ${ }^{1 *}$, Christopher J Millett ${ }^{1,2}$, Preet K Dhillon', SV Subramanian ${ }^{3}$ and Shah Ebrahim ${ }^{1,4}$
}

\begin{abstract}
Background: To investigate the prevalence of obesity and diabetes among adult men and women in India consuming different types of vegetarian diets compared with those consuming non-vegetarian diets.

Methods: We used cross-sectional data of 156,317 adults aged 20-49 years who participated in India's third National Family Health Survey (2005-06). Association between types of vegetarian diet (vegan, lacto-vegetarian, lacto-ovo vegetarian, pesco-vegetarian, semi-vegetarian and non-vegetarian) and self-reported diabetes status and measured body mass index (BMI) were estimated using multivariable logistic regression adjusting for age, gender, education, household wealth, rural/urban residence, religion, caste, smoking, alcohol use, and television watching.

Results: Mean BMI was lowest in pesco-vegetarians $\left(20.3 \mathrm{~kg} / \mathrm{m}^{2}\right)$ and vegans $\left(20.5 \mathrm{~kg} / \mathrm{m}^{2}\right)$ and highest in lacto-ovo vegetarian $\left(21.0 \mathrm{~kg} / \mathrm{m}^{2}\right)$ and lacto-vegetarian $\left(21.2 \mathrm{~kg} / \mathrm{m}^{2}\right)$ diets. Prevalence of diabetes varied from $0.9 \%(95 \% \mathrm{Cl}$ : 0.8-1.1) in person consuming lacto-vegetarian, lacto-ovo vegetarian (95\% Cl:0.6-1.3) and semi-vegetarian (95\% Cl:0.7-1.1) diets and was highest in those persons consuming a pesco-vegetarian diet (1.4\%; 95\% Cl:1.0-2.0). Consumption of a lacto- (OR:0.67;95\% Cl:0.58-0.76; $<<0.01$ ), lacto-Ovo (OR:0.70; 95\% Cl:0.51-0.96; $=0.03$ ) and semi-vegetarian (OR:0.77; $95 \%$ Cl:0.60-0.98; $p=0.03$ ) diet was associated with a lower likelihood of diabetes than a non-vegetarian diet in the adjusted analyses.

Conclusions: In this large, nationally representative sample of Indian adults, lacto-, lacto-ovo and semi-vegetarian diets were associated with a lower likelihood of diabetes. These findings may assist in the development of interventions to address the growing burden of overweight/obesity and diabetes in Indian population. However, prospective studies with better measures of dietary intake and clinical measures of diabetes are needed to clarify this relationship.
\end{abstract}

Keywords: Vegetarian diets, Diabetes, Obesity, Men, Women, NFHS-3, India

\section{Background}

Studies from Western countries suggest that vegetarian diets may have a protective role against the development of obesity and diabetes [1-5]. The European Prospective Investigation Study (EPIC-Oxford) found that mean BMI was highest in meat-eaters, lowest in vegans, and intermediate in fish-eaters and vegetarians [6]. In the Nurses' Health Study, intake of red meat and processed meats were associated with increased risk of diabetes [7]. In Seventh-day Adventist cohort studies initiated in the 1960s-1970s, diabetes was less prevalent in vegetarian

\footnotetext{
* Correspondence: sutapa.agrawal@phfi.org

'South Asia Network for Chronic Disease, Public Health Foundation of India, Fourth Floor, Plot no 47, Sector 44, Gurgaon (Haryana)-122002, New Delhi, India

Full list of author information is available at the end of the article
}

than in semi-vegetarian (those who ate fish and poultry, but $<1$ time/wk)_ or non-vegetarian church-goers and processed meat eaters [5,7-9]. These observational findings are also supported by experimental data which have shown that the selection of foods found in vegetarian diets may carry metabolic advantages for the prevention of type 2 diabetes [10].

India is experiencing an alarming increase in the prevalence of type 2 diabetes [10-15]. The resulting morbidity, economic costs, reduced quality of life, and risk for complications make preventive strategies imperative. The contribution of the Indian diet to the increasing prevalence of diabetes in the country is not well understood. Within this, there is little information on whether the vegetarian diet confers a similar protective effect 
against obesity and diabetes that have been demonstrated in western studies. This is an important question given the ongoing preponderance of vegetarianism in certain social and religious groups in India coupled with an increase in meat eating associated with growth in western-style diets in some section of the Indian society. Moreover, vegetarianism in India is associated with unique characteristics. It is usually a lifelong pattern and adherence crosses multiple generations; it generally comprises high consumption of whole grains, legumes, nuts and seeds and dairy with spices and seasonings unique to the Indian diet. Hence, the combination/or the pattern of vegetarian diet may yield different findings than similar studies conducted in the West and it is thus possible to assess dietary associations with chronic diseases which have been difficult in the West due to low frequency. This study uses data from the third National Family Health Survey (NFHS-3, 2005-06), a survey of 109,041 Indian households which collected information on a wide range of dietary, societal, lifestyle, and environmental determinants of morbidity and chronic ailments including diabetes [16]. The NFHS-3 provides a unique opportunity to examine associations between types of vegetarian diet and diabetes and obesity in a large, nationally representative sample. We hypothesized that exclusively vegetarian diets, such as vegan, lacto- or lacto-ovo vegetarian, are associated with a lower prevalence of diabetes and obesity compared with a non-vegetarian diet.

\section{Methods}

\section{Data and study setting}

We used cross-sectional data from India's third National Family Health Survey (NFHS-3, 2005-06) conducted in 29 states which comprises more than $99 \%$ of the country's population, but excluded the Union Territories. Details of survey objectives and survey methods including sampling frame and questionnaires are provided elsewhere [16]. Briefly, this survey was designed on the lines of the Demographic and Health Surveys (DHS) (available at www.measuredhs.com) that have been conducted in many developing countries since the 1980s and in India the survey was designed to provide estimates of key indicators (except HIV prevalence) for each state by urban and rural areas. The NFHS has been conducted in India for successive three rounds, each at an interval of 5 years. NFHS-3 is the most recent major national health survey in India that collected demographic, socioeconomic and health information from a nationally representative probability sample of 124,385 women (62.6\%) aged 15-49 years and 74,369 men (37.4\%) aged 15-54 years residing in 109,041 households. The data was obtained at the individual level by face-to-face interviews conducted in the respondents' homes.
The NFHS-3 samples were geo-coded to the primary sampling unit, district, and state to which they belonged. A uniform multistage sampling strategy was adopted in all the states, with separate sampling in urban and rural areas. In rural areas, a two-stage sample was carried out using a list of villages from the 2001 census as the sampling frame. In the first stage, a stratified sample of villages was drawn with probability proportional to the size of the village. In the second stage, a random selection of households was drawn in each village from a complete list of households compiled during field visits carried out in each sampled village. In urban areas, a similar procedure was implemented beginning with a stratified random sample of municipal wards based on the 2001 census. Further, one census enumeration block (about 150-200 households) was selected from within the wards using probability proportional to size sampling frame. Finally, as in rural areas, field enumerators undertook a house listing operation in selected blocks and a random sample of households was made. In both rural and urban areas, 30 households were targeted for selection in each of the sampled units.

The overall household response rate in NFHS-3 was 98\%. All women aged 15-49 years in selected households were invited to participate in the National Family Health Survey. Interviews were conducted in one of the 18 Indian languages in the respondent's home and the response rates were $95 \%$ for women and $87 \%$ for men. During interviews, the weights and heights of survey respondents were measured and blood sample were drawn by trained field technicians using standardised measuring equipment designed for survey settings in developing country.

The analysis we present here is restricted to 156,317 sample population comprising of 99,574 women and 56,742 men aged 20-49 years living in the sample households. We excluded age below 20 years to avoid any cases of childhood diabetes for which the etiology and risk factors might be different. Age above 50 years is also excluded (for men only) for comparison purpose since information for women age above 50 years is not collected in the survey.

\section{Outcome evaluation}

The survey asked participants the question, 'Do you currently have diabetes?'. However, neither data on physician-reported diagnosis of diabetes nor data on fasting blood glucose was available in the NFHS-3 to verify a self-report.

\section{Assessment of dietary intake}

In NFHS-3, consumption of selected food item was assessed by asking, 'How often do you yourself consume the following food items: daily, weekly, occasionally or never?' 
related to the consumption of milk or curd, pulses or beans, dark green leafy vegetables, fruits, eggs, fish, chicken or meat. Based on the frequency of consumption, vegetarian status $[8,17,18]$ was categorized by defining vegans as subjects who reported never consuming animal products (chicken or meat, fish, eggs, milk or curd); lacto-vegetarian as those who reported consuming fruits, vegetables, pulses or beans, milk or curd, either daily, weekly or occasionally but no fish, eggs or chicken or meat; lacto-ovo vegetarian as those who reported consuming fruits, vegetables, pulses or beans, milk or curd, and or eggs either daily, weekly or occasionally but no fish or chicken or meat; pescovegetarian: who reported consuming fruits, vegetables, pulses or beans, milk or curd, and or eggs or fish either daily, weekly or occasionally but no chicken or meat; semivegetarian: who reported consuming fruits, vegetables, pulses or beans, animal products (chicken or meat, eggs, milk or curd) either daily, weekly or occasionally but no fish; non-vegetarian: who reported consuming fruits, vegetables, pulses or beans, animal products (chicken or meat, fish, eggs, milk or curd) either daily, weekly or occasionally.

\section{Other predictor variables and covariates}

The survey collected information on demographic, socioeconomic factors, lifestyle factors and anthropometric measurements. Respondents were weighed using a solar powered digital scale (SECA 874 digital scale ${ }^{\text {a)}}$ ) with an accuracy of $\pm 100 \mathrm{~g}$ [19]. Their height was measured using an adjustable wooden measuring board, specifically designed to provide accurate measurements (to the nearest $0.1 \mathrm{~cm}$ ) [19]. Indian adult population standard [20-22] categories of Body Mass Index (BMI, $\mathrm{kg} / \mathrm{m}^{2}$ ) were used: $\leq 18.5 \mathrm{~kg} / \mathrm{m}^{2}$ (underweight); 18.5 to $22.9 \mathrm{~kg} / \mathrm{m}^{2}$ (normal), 23.0 to $24.9 \mathrm{~kg} / \mathrm{m}^{2}$ (overweight), and $\geq 25.0 \mathrm{~kg} / \mathrm{m}^{2}$ (obese). The information on exposure to tobacco smoke wasyes-active smoking (person currently smokes) and no smoking (the person has never smoked). Information on past smoking was not available in the dataset. Use of alcohol was quantified as ever drinker (drinks taken almost every day or about once weekly or less than once weekly) and never drinker. Frequency of watching television (almost every day, at least once weekly, less than once weekly, not at all) was used as a measure of sedentary behavior. Other covariates in our analysis include: age categories (20-29, 30-39, 40-49 years); gender, education (no education, primary complete, middle complete, higher and above); religion (Hindu, Muslim, Christian, Sikh, Others); caste/tribe (scheduled caste, scheduled tribe, other backward class, others, missing caste); wealth quintiles (based on 33 assets and housing characteristics graded lowest, second, middle, fourth, highest); and place of residence (urban, rural). For detailed definition of some variables and items used for construction of the wealth index, see Table 1.

\section{Statistical analysis}

As certain states and certain groups of respondents were oversampled, sample weights were used to restore the representativeness of the sample [16]. Descriptive statistics were calculated with the use of standard methods (such as frequencies and percentages) for the total sample $(\mathrm{n}=156,317)$. Differences in proportions for categorical variables were tested using Pearson's $X^{2}$ tests. Trend tests were also carried out scoring the variables in different categories by using likelihood ratio tests. Multivariable logistic regression models were used to estimate the odds ratios of types of vegetarian diet intake on self-reported diabetes after controlling for potential confounders and also examining the independent effects of other risk factors. Both unadjusted and adjusted models were constructed with 95\% confidence intervals to account for potential confounders and mediators. Model 1 presents unadjusted results; Model 2 presents results adjusted for BMI, lifestyle factors and socio-demographic factors which may be confounders to exhibit any independent effect of vegetarian diet on diabetes prevalence; Model 3 presents results adjusted for all the above factors except BMI. Results are presented in the form of odds ratios (ORs) with 95\% confidence intervals $(95 \% \mathrm{CI})$. The estimation of confidence intervals takes into account the design effects due to clustering at the level of the primary sampling unit. Before carrying out the models, we tested for the possibility of multicolinearity between the variables. In the correlation matrix, all pair wise Pearson correlation coefficients are $<0.5$, suggesting that multicolinearity is not a problem. All the analysis including the logistic regression models were conducted using the SPSS statistical software package, version 19 (IBM SPSS Statistics, Chicago, IL, USA).

As the effect of type of vegetarian diet consumption on the prevalence of diabetes are likely to vary by sex, due to the large gender differences in nutritional status ${ }^{b}$ in India, the susceptibility to disease, and access to treatment and care in a developing country in general, an analysis was also carried out for men and women separately.

\section{Ethics statement}

The data were analyzed anonymously, using publicly available secondary data, therefore no ethics review is required for this work. The National Family Health Survey was approved by the ethical review boards of the implementing agencies and the Indian government. Participation in the survey was totally voluntary. The survey obtained written informed consent from each respondent (men and women) before asking questions, and separately before obtaining height and weight measurements. 
Table 1 Sample distribution and percentage prevalence of diabetes among men $(n=56,742)$ and women $(n=99,574)$ age $20-49$ years according to intake of specific food items in the national family health survey, India 2005-06

\begin{tabular}{|c|c|c|c|c|c|c|c|c|c|}
\hline \multirow[t]{2}{*}{ Frequency of intake } & \multicolumn{3}{|l|}{ Men } & \multicolumn{3}{|l|}{ Women } & \multicolumn{3}{|l|}{ Total } \\
\hline & Total N [\%] & $\begin{array}{l}\text { Diabetes cases } \\
\mathrm{N}[\%]\end{array}$ & $x^{2} p$ value & $\begin{array}{l}\text { Subjects } \\
\mathrm{N}[\%]\end{array}$ & $\begin{array}{l}\text { Diabetes cases } \\
\mathrm{N}[\%]\end{array}$ & $x^{2} p$ value & Total N [\%] & $\begin{array}{l}\text { Diabetes cases } \\
\mathrm{N}[\%]\end{array}$ & $x^{2} p$ value \\
\hline Milk or curd & & & $<0.0001$ & & & $<0.0001$ & & & $<0.0001$ \\
\hline Daily & $26307[46.4]$ & $391[1.5]$ & & $40366[40.5]$ & $492[1.2]$ & & $66673[42.7]$ & $883[1.3]$ & \\
\hline Weekly & $11554[20.4]$ & $117[1.0]$ & & $15071[15.1]$ & $138[0.9]$ & & $26626[17.0]$ & $255[1.0]$ & \\
\hline Occasionally & $14757[26.0]$ & $138[0.9]$ & & 32918 [33.1] & $302[0.9]$ & & $47675[30.5]$ & $440[0.9]$ & \\
\hline Never & $4114[7.3]$ & $74[1.8]$ & & $11202[11.3]$ & $117[1.0]$ & & $15317[9.8]$ & $191[1.2]$ & \\
\hline Pulses and beans & & & $<0.0001$ & & & $<0.0001$ & & & $<0.0001$ \\
\hline Daily & $29863[52.6]$ & $437[1.5]$ & & $52440[52.7]$ & $538[1.0]$ & & $82303[52.7]$ & $975[1.2]$ & \\
\hline Weekly & $21705[38.3]$ & $219[1.0]$ & & $36597[36.8]$ & $360[1.0]$ & & $58302[37.3]$ & $579[1.0]$ & \\
\hline Occasionally & $4660[8.2]$ & $51[1.1]$ & & 9663 [9.7] & $131[1.4]$ & & $14323[9.2]$ & $182[1.3]$ & \\
\hline Never & $505[0.9]$ & $13[2.6]$ & & $852[0.9]$ & $20[2.3]$ & & $1357[0.9]$ & $33[2.4]$ & \\
\hline Green leafy vegetables & & & 0.149 & & & 0.090 & & & 0.368 \\
\hline Daily & $33982[59.9]$ & $453[1.3]$ & & $64095[64.4]$ & $674[1.1]$ & & $98076[62.7]$ & $1127[1.1]$ & \\
\hline Weekly & $19270[34.0]$ & $231[1.2]$ & & 28606 [28.7] & $286[1.0]$ & & $47876[30.6]$ & $517[1.1]$ & \\
\hline Never/ Occasionally & $3480[6.1]$ & $35[1.0]$ & & $6840[6.9]$ & $89[1.3]$ & & $10321[6.6]$ & $125[1.2]$ & \\
\hline Fruits & & & $<0.0001$ & & & $<0.0001$ & & & $<0.0001$ \\
\hline Daily & 7320 [12.9] & 125 [1.7] & & 12789 [12.9] & 206 [1.6] & & 20109 [12.9] & 331 [1.6] & \\
\hline Weekly & 19368 [34.1] & 255 [1.3] & & 26731 [26.9] & 276 [1.0] & & 46099 [29.5] & 531 [1.2] & \\
\hline Occasionally & $28484[50.2]$ & 296 [1.0] & & $56336[56.6]$ & $503[0.9]$ & & 84820 [54.3] & $800[0.9]$ & \\
\hline Never & 1546 [2.7] & $44[2.8]$ & & 3631 [3.6] & $63[1.7]$ & & 5177 [3.3] & 107 [2.1] & \\
\hline Eggs & & & $<0.0001$ & & & $<0.0001$ & & & $<0.0001$ \\
\hline Daily & $2931[5.2]$ & $56[1.9]$ & & 3475 [3.5] & $60[1.7]$ & & 6405 [4.1] & 115 [1.8] & \\
\hline Weekly & 20682 [36.5] & 317 [1.5] & & 28778 [28.9] & 363 [1.3] & & 49460 [31.6] & $680[1.4]$ & \\
\hline Occasionally & 19786 [34.9] & 201 [1.0] & & 32635 [32.8] & 287 [0.9] & & 52421 [33.5] & 488 [0.9] & \\
\hline Never & 13330 [23.5] & $146[1.1]$ & & 34647 [34.8] & 340 [1.0] & & $47977[30.7]$ & $486[1.0]$ & \\
\hline Fish & & & $<0.0001$ & & & $<0.0001$ & & & $<0.0001$ \\
\hline Daily & 3706 [6.5] & $90[2.4]$ & & 6505 [6.5] & 149 [2.3] & & $10211[6.5]$ & $240[2.4]$ & \\
\hline Weekly & $14414[25.4]$ & 238 [1.7] & & 22070 [22.2] & 304 [1.4] & & 36484 [23.3] & $542[1.5]$ & \\
\hline
\end{tabular}


Table 1 Sample distribution and percentage prevalence of diabetes among men $(n=56,742)$ and women $(n=99,574)$ age $20-49$ years according to intake of specific food items in the national family health survey, India 2005-06 (Continued)

\begin{tabular}{|c|c|c|c|c|c|c|c|c|c|}
\hline Occasionally & $21818[38.5]$ & $225[1.0]$ & & $34242[34.4]$ & $264[0.8]$ & & $56060[35.9]$ & $489[0.9]$ & \\
\hline Never & $16782[29.6]$ & $167[1.0]$ & & 36724 [36.9] & $331[0.9]$ & & 53506 [34.2] & $498[0.9]$ & \\
\hline Chicken or meat & & & $<0.0001$ & & & $<0.0001$ & & & $<0.0001$ \\
\hline Daily & $706[1.2]$ & $6[0.9]$ & & $839[0.8]$ & $14[1.7]$ & & 1545 [1.0] & $20[1.3]$ & \\
\hline Weekly & $15609[27.5]$ & 269 [1.7] & & 21938 [22.0] & $292[1.3]$ & & 37548 [24.0] & $561[1.5]$ & \\
\hline Occasionally & $26135[46.1]$ & 291 [1.1] & & $42222[42.0]$ & $423[1.0]$ & & 68357 [43.7] & $714[1.0]$ & \\
\hline Never & $14272[25.2]$ & 155 [1.1] & & $34537[34.7]$ & $320[0.9]$ & & 48809 [31.2] & $475[1.0]$ & \\
\hline
\end{tabular}




\section{Results}

Types of vegetarian diet consumption in India and states The sample distribution and percentage prevalence of diabetes among men $(n=56,742)$ and women $(n=99,574)$ aged 20-49 years according to intake of specific food items in the National Family Health Survey, India 200506 is presented in Table 1. Table 2 gives the percentage consumption of different types of vegetarian diet among adult population $(n=156,317)$ age $20-49$ years in India and states. Overall a majority (two-third- 64\%) of the sample population eat a non-vegetarian diet either daily, weekly or at least occasionally where as one-fourth is lacto-vegetarian (Table 1). Other dietary patterns are followed by a relatively smaller percentage of Indian population: semi-vegetarian-5.2\%, lacto-ovo vegetarian-3.2\%, pesco-vegetarian-2.2\% and vegan-1.6\%. More than $80 \%$ of the population consume a non-vegetarian diet in northeastern region, in southern region (except the state of Karnataka), most of the states in eastern region (except Bihar), and the western state of Goa. More than half the population in the northern states of Punjab, Haryana, Rajasthan, and in the western state of Gujarat follow a lacto-vegetarian diet. One in five people in Jammu and Kashmir follow a semi-vegetarian diet (without fish) whereas one in ten people in Goa (11.8\%) and Manipur (9.0\%), $7.8 \%$ in Tripura, $7.2 \%$ in Orissa, $5.2 \%$ in Kerala and $4.1 \%$ in West Bengal consume pesco-vegetarian diet (dominated by fish). In the state of Delhi, one out of ten people is a lacto-ovo vegetarian where as the western states of Gujarat (4.9\%) and Maharashtra (4.0\%) have the highest percentages of vegans.

\section{Distribution of self reported diabetes cases and diabetes prevalence by study covariates}

Among those who reported diabetes, three out of five were aged between 40-49 years, a majority (59\%) were women, two out of five had a secondary education, three-fourth follow Hindu religion, two out of five belonged to general caste, two out of five belonged to household with a highest wealth status, a majority don't smoke or drink alcohol, more than half the participants watched TV almost every day and a third were obese (Table 3 ).

The overall prevalence of diabetes was significantly higher $(\mathrm{p}<0.0001)$ among men $(1.3 \%)$ than among women $(0.9 \%)$ (Table 3). Significant associations between age and diabetes prevalence were observed, diabetes being more prevalent $(2.8 \%)$ in the highest age group (40-49 years). Diabetes prevalence increased according to household wealth status and was almost double in urban population compared with their rural counterparts (1.6 vs 0.8) and highest among those with a higher secondary and above education (all p < 0.0001). Prevalence of diabetes was also higher among those who were currently smoking tobacco $(1.3 \%)$ or ever consumed alcohol (1.4\%), who were either overweight
(2.1\%) or obese $(3.2 \%)$ and those who watched television almost every day (1.4\%).

\section{Prevalence of diabetes and obesity according to types of vegetarian diet consumption}

Table 4 gives the unadjusted prevalence of diabetes and obesity by types of vegetarian diet consumption. No apparent trend in diabetes prevalence based on type of vegetarian diet was found ( $\mathrm{p}$ for trend $=0.002$ ). Prevalence of diabetes varied from $0.9 \%$ (95\% CI:0.8-1.1) each in lactovegetarian, lacto-ovo vegetarian (95\% CI:0.6-1.3) and semi-vegetarian (95\% CI:0.7-1.1) to $1.0 \%$ in vegan (95\% CI: $0.6-1.7), 1.2 \%$ (95\% CI:1.1-1.3) in non-vegetarian and highest in pesco-vegeterian diets (1.4\%; 95\% CI:1.0-2.0). The range between the lowest and highest BMIs for all groups were reasonably low (less than $1 \mathrm{~kg} / \mathrm{m}^{2}$ ). Mean BMI was $20.3 \mathrm{~kg} / \mathrm{m}^{2}$ in pesco-vegetarians and $20.5 \mathrm{~kg} / \mathrm{m}^{2}$ in vegans, $20.6 \mathrm{~kg} / \mathrm{m}^{2}$ in semi-vegetarians, $20.7 \mathrm{~kg} / \mathrm{m}^{2}$ in nonvegetarians, $21.0 \mathrm{~kg} / \mathrm{m}^{2}$ in lacto-ovo vegetarians and $21.2 \mathrm{~kg} / \mathrm{m}^{2}$ in lacto-vegetarians. For BMIs $\geq 23 \mathrm{~kg} / \mathrm{m}^{2}$, the prevalence of diabetes was $1.7 \%$ in lacto-ovo vegetarians, $2.0 \%$ in semi-vegetarians, $2.1 \%$ in lacto-vegetarians, $2.6 \%$ in pesco-vegetarians, $2.8 \%$ in vegans, and $2.9 \%$ in nonvegetarians (data not shown). For BMIs $\geq 30 \mathrm{~kg} / \mathrm{m}^{2}$, the prevalence of diabetes was $2.1 \%$ in lacto-ovo vegetarians, $3.7 \%$ in lacto-vegetarians, $3.8 \%$ in semi-vegetarians, $5.2 \%$ in vegans, $5.3 \%$ in pesco-vegetarians and $5.4 \%$ in nonvegetarians (data not shown).

\section{Vegetarian diet consumption according to non-dietary variables}

Table 5 shows the vegetarian diet consumption by nondietary variables. Socioeconomic demographic and lifestyle characteristics differed substantially $(\mathrm{p}<0.001)$ among the dietary groups but overall non-vegetarian diet was predominant in all socio economic and demographic categories followed by lacto-vegetarian diet.

Association between type of vegetarian diet and diabetes In multivariable logistic regression analysis (Table 6), after adjustment for age, gender, education, household wealth, rural/urban residence, religion, caste, smoking, alcohol use, television watching and body mass index, consumption of lacto-vegetarian (AOR:0.67; 95\% CI:0.58-0.76), lacto-ovo vegetarian (AOR:0.69; 95\% CI:0.50-0.95) and semi-vegetarian (AOR:0.76; 95\% CI: 0.60-0.98) diets were associated with a lower likelihood of diabetes than nonvegetarian diet. The association remained almost unchanged when BMI was removed from the analyses.

\section{Association between type of vegetarian diet and diabetes stratified by sex}

To examine the sex differences in the adjusted effect of vegetarian diet on diabetes prevalence, we also carried 
Table 2 Percentage consumption of different types of diet among adult population age 20-49 years in India and states, NFHS-3, 2005-06

\begin{tabular}{|c|c|c|c|c|c|c|c|}
\hline \multirow[t]{2}{*}{ India/States } & \multicolumn{6}{|c|}{ Types of diet } & \multirow[t]{2}{*}{ Total N } \\
\hline & $\begin{array}{l}\text { Vegan } \\
\mathrm{N}[\%]\end{array}$ & $\begin{array}{l}\text { Lacto-vegetarian } \\
\mathrm{N}[\%]\end{array}$ & $\begin{array}{l}\text { Lacto-ovo vegetarian } \\
\mathrm{N}[\%]\end{array}$ & $\begin{array}{l}\text { Pesco- vegetarian } \\
N[\%]\end{array}$ & $\begin{array}{l}\text { Semi-vegetarian } \\
\mathrm{N}[\%]\end{array}$ & $\begin{array}{l}\text { Non-vegetarian } \\
\mathrm{N}[\%]\end{array}$ & \\
\hline India & $2560[1.6]$ & $37797[24.2]$ & $5002[3.2]$ & $3446[2.2]$ & $8140[5.2]$ & 99372 [63.6] & 156317 \\
\hline \multicolumn{8}{|l|}{ Northern region } \\
\hline Jammu \& Kashmir & $9[0.6]$ & $276[18.4]$ & $18[1.2]$ & $9[0.6]$ & $297[19.8]$ & $891[59.4]$ & 1500 \\
\hline Himachal Pradesh & $17[1.8]$ & $429[45.6]$ & $76[8.1]$ & $13[1.4]$ & $137[14.6]$ & $269[28.6]$ & 941 \\
\hline Punjab & $138[3.4]$ & $2149[52.3]$ & $275[6.7]$ & $13[0.3]$ & $420[10.2]$ & $1112[27.1]$ & 4107 \\
\hline Uttaranchal & $20[1.6]$ & $324[26.6]$ & $84[6.9]$ & $15[1.2]$ & 108 [8.9] & $669[54.8]$ & 1220 \\
\hline Haryana & $107[3.5]$ & 2099 [68.9] & $205[6.7]$ & $6[0.2]$ & $148[4.9]$ & $482[15.8]$ & 3047 \\
\hline Delhi & $43[2.1]$ & $645[30.9]$ & 222 [10.6] & $25[1.2]$ & $192[9.2]$ & $963[46.1]$ & 2090 \\
\hline Rajasthan & $236[2.9]$ & $5060[62.1]$ & $393[4.8]$ & $62[0.8]$ & 869 [10.7] & $1528[18.8]$ & 8148 \\
\hline \multicolumn{8}{|l|}{ Central region } \\
\hline Uttar Pradesh & $264[1.2]$ & 8458 [37.7] & $1227[5.5]$ & $336[1.5]$ & 835 [3.7] & $11343[50.5]$ & 22463 \\
\hline Chhattisgarh & $69[2.1]$ & $484[14.5]$ & $101[3.0]$ & $60[1.8]$ & $57[1.7]$ & $2574[77.0]$ & 3345 \\
\hline Madhya Pradesh & $294[3.1]$ & 3975 [42.2] & $479[5.1]$ & $223[2.4]$ & 463 [4.9] & 3980 [42.3] & 9414 \\
\hline \multicolumn{8}{|l|}{ Eastern region } \\
\hline Bihar & $50[0.5]$ & 1812 [17.3] & $66[0.6]$ & $382[3.6]$ & $120[1.1]$ & 8037 [76.8] & 10467 \\
\hline West Bengal & $43[0.3]$ & $183[1.4]$ & $16[0.1]$ & $554[4.1]$ & $94[0.7]$ & $12548[93.4]$ & 13438 \\
\hline Jharkhand & $49[1.3]$ & $214[5.5]$ & $37[1.0]$ & $80[2.1]$ & $81[2.1]$ & 3395 [88.0] & 3856 \\
\hline Orissa & $50[0.8]$ & $225[3.8]$ & $19[0.3]$ & $432[7.2]$ & $66[1.1]$ & $5168[86.7]$ & 5960 \\
\hline \multicolumn{8}{|c|}{ Northeastern region } \\
\hline Sikkim & $0[0.0]$ & 9 [9.6] & $1[1.1]$ & $1[1.1]$ & $6[6.4]$ & 77 [81.9] & 94 \\
\hline Arunachal Pradesh & $0[0.0]$ & $2[1.3]$ & $1[0.6]$ & $2[1.3]$ & $2[1.3]$ & 152 [95.6] & 159 \\
\hline Nagaland & $0[0.0]$ & $1[0.5]$ & $0[0.0]$ & $1[0.5]$ & $2[1.0]$ & $204[98.1]$ & 208 \\
\hline Manipur & $1[0.3]$ & $1[0.3]$ & $0[0.0]$ & $31[9.0]$ & $3[0.9]$ & $307[89.5]$ & 343 \\
\hline Mizoram & $0[0.0]$ & $0[0.0]$ & $1[0.7]$ & $1[0.7]$ & $6[4.3]$ & $131[94.2]$ & 139 \\
\hline Tripura & $1[0.2]$ & $4[0.7]$ & $1[0.2]$ & $46[7.8]$ & $2[0.3]$ & $536[90.8]$ & 590 \\
\hline Meghalaya & $0[0.0]$ & $3[0.8]$ & $1[0.3]$ & $9[2.3]$ & $5[1.3]$ & $371[95.4]$ & 389 \\
\hline Assam & $5[0.1]$ & $72[1.6]$ & $13[0.3]$ & $132[3.0]$ & $12[0.3]$ & $4135[94.6]$ & 4369 \\
\hline \multicolumn{8}{|l|}{ Western region } \\
\hline Gujarat & $400[4.9]$ & 4546 [55.6] & $342[4.2]$ & 159 [1.9] & $399[4.9]$ & 2330 [28.5] & 8176 \\
\hline Maharashtra & $643[4.0]$ & 3614 [22.7] & 529 [3.3] & $1.35[0.8]$ & 912 [5.7] & 10068 [63.3] & 15901 \\
\hline Goa & $3[1.2]$ & $10[3.9]$ & $2[0.8]$ & 30 [11.8] & $2[0.8]$ & 207 [81.5] & 254 \\
\hline \multicolumn{8}{|l|}{ Southern region } \\
\hline Andhra Pradesh & $45[0.4]$ & $579[4.7]$ & $222[1.8]$ & $78[0.6]$ & 1129 [9.1] & $10299[83.4]$ & 12352 \\
\hline Karnataka & $41[0.4]$ & 2126 [22.2] & $385[4.0]$ & $134[1.4]$ & 979 [10.2] & 5932 [61.8] & 9597 \\
\hline Kerala & $11[0.2]$ & $81[1.8]$ & $37[0.8]$ & $234[5.2]$ & $54[1.2]$ & $4045[90.7]$ & 4462 \\
\hline Tamil Nadu & $21[0.2]$ & $416[4.5]$ & $249[2.7]$ & $243[2.6]$ & 740 [8.0] & 7619 [82.0] & 9288 \\
\hline
\end{tabular}

out separate analyses for men and women (Table 7). The likelihood of having a positive diabetes status was significantly lower among men following a lacto-vegetarian (AOR:0.66; 95\% CI:0.52-0.82;p < 0.0001) and semi-vegetarian $\operatorname{diet}($ AOR:0.45; 95\% CI:0.29-0.71;p = 0.001) while only lactovegetarian diet (AOR:0.70; 95\% CI:0.59-0.82;p <0.0001) consumption was associated with a lower likelihood of diabetes among women.

\section{Discussion}

This cross-sectional, population-based study adds to the limited data on associations between type of vegetarian 
Table 3 Percentage distribution of participants by self reported diabetes status and prevalence of diabetes according to non-dietary variables, India NFHS 2005-06

\begin{tabular}{|c|c|c|c|c|c|c|}
\hline \multirow[t]{2}{*}{ Characteristics } & \multirow{2}{*}{$\begin{array}{l}\text { Total participants } \\
\mathrm{N}[\%]^{*}\end{array}$} & \multicolumn{2}{|c|}{ Diabetes cases } & \multirow[t]{2}{*}{$x^{2} P$ values } & \multirow{2}{*}{$\begin{array}{l}\text { Diabetes prevalence } \\
\mathrm{N}[\%]\end{array}$} & \multirow[t]{2}{*}{$x^{2} \mathrm{P}$ values } \\
\hline & & Reported N [\%] & Not reported N [\%] & & & \\
\hline $\mathrm{N}[\%]$ & 156,317 & $1,769[1.1]$ & $154,501[98.9]$ & & & \\
\hline Age & & & & $<0.0001$ & & $<0.0001$ \\
\hline $20-29 y$ & $66,038[42.2]$ & 204 [11.5] & $65807[42.6]$ & & 0.3 & \\
\hline $30-39 y$ & $52,567[33.6]$ & $520[29.4]$ & 5203833.7] & & 1.0 & \\
\hline $40-49 y$ & $37,711[24.1]$ & $1045[59.1]$ & $36656[23.7]$ & & 2.8 & \\
\hline Sex & & & & $<0.0001$ & & $<0.0001$ \\
\hline Men & $56,742[36.3]$ & $994[40.7]$ & $73367[36.3]$ & & 1.3 & \\
\hline Women & $99,574[63.7]$ & $1096[59.3]$ & $123244[63.7]$ & & 0.9 & \\
\hline Education $^{a}$ & & & & $<0.0001$ & & $<0.0001$ \\
\hline No education & $56,720[36.3]$ & $529[27.3]$ & $63709[36.4]$ & & 0.8 & \\
\hline Primary & $24,493[15.7]$ & $350[17.1]$ & $30593[15.7]$ & & 1.1 & \\
\hline Secondary & $58,448[37.4]$ & $909[42.6]$ & $84284[37.3]$ & & 1.1 & \\
\hline Higher and above & $16,639[10.6]$ & 302 [13.0] & $18001[10.6]$ & & 1.7 & \\
\hline Caste/tribe ${ }^{b}$ & & & & $<0.0001$ & & $<0.0001$ \\
\hline Scheduled caste & $29831[18.5]$ & $350[17.2]$ & $36736[18.5]$ & & 0.9 & \\
\hline Scheduled tribe & $12734[8.1]$ & $75[3.1]$ & $16105[8.2]$ & & 0.5 & \\
\hline Other backward class & $60977[39.0]$ & 728 [35.3] & $77187[39.0]$ & & 0.9 & \\
\hline General & $48854[31.3]$ & 840 [39.9] & $60489[31.2]$ & & 1.4 & \\
\hline Missing caste & $4821[3.1]$ & $90[4.6]$ & $5450[3.1]$ & & 1.6 & \\
\hline Religion ${ }^{c}$ & & & & $<0.0001$ & & $<0.0001$ \\
\hline Hindu & $127375[81.5]$ & 1616 [77.3] & 159511 [81.5] & & 1.0 & \\
\hline Muslim & $19781[12.7]$ & 311 [15.1] & $25806[12.6]$ & & 1.2 & \\
\hline Christian & $3816[2.4]$ & $86[4.2]$ & $4657[2.4]$ & & 1.8 & \\
\hline Sikh & $2845[1.8]$ & $48[2.1]$ & $3534[1.8]$ & & 1.3 & \\
\hline Others & $2500[1.6]$ & $28[1.2]$ & $3104[1.6]$ & & 0.8 & \\
\hline Wealth quintiles $^{d}$ & & & & $<0.0001$ & & $<0.0001$ \\
\hline Lowest & $26389[16.9]$ & $171[8.0]$ & $33269[17.0]$ & & 0.5 & \\
\hline Second & $28751[18.4]$ & 270 [13.6] & $36780[18.4]$ & & 0.7 & \\
\hline Middle & $31232[20.0]$ & $272[13.1]$ & $39975[20.1]$ & & 0.7 & \\
\hline Fourth & $33560[21.5]$ & $490[24.2]$ & $42082[21.4]$ & & 1.2 & \\
\hline Highest & $36385[23.3]$ & $887[41.0]$ & $44505[23.1]$ & & 2.0 & \\
\hline Place of residence & & & & $<0.0001$ & & $<0.0001$ \\
\hline Urban & $54134[34.6]$ & $1068[50.8]$ & $66879[34.4]$ & & 1.6 & \\
\hline Rural & $102183[65.4]$ & $1022[49.2]$ & $129732[65.6]$ & & 0.8 & \\
\hline Body Mass Index $\left(\mathrm{kg} / \mathrm{m}^{2}\right)^{\mathrm{e}}$ & & & & $<0.0001$ & & $<0.0001$ \\
\hline$\leq 18.5 \mathrm{~kg} / \mathrm{m}^{2}$ & $46021[30.9]$ & $694[14.6]$ & $85097[31.1]$ & & 0.8 & \\
\hline $18.5-22.9 \mathrm{~kg} / \mathrm{m}^{2}$ & $67836[45.5]$ & $295[33.2]$ & $64754[45.7]$ & & 0.5 & \\
\hline $23.0-24.9 \mathrm{~kg} / \mathrm{m}^{2}$ & 15089 [10.1] & $347[16.4]$ & $16537[10.1]$ & & 2.1 & \\
\hline$\geq 25.0 \mathrm{~kg} / \mathrm{m}^{2}$ & $20050[13.5]$ & $691[35.8]$ & $20858[13.2]$ & & 3.2 & \\
\hline
\end{tabular}


Table 3 Percentage distribution of participants by self reported diabetes status and prevalence of diabetes according to non-dietary variables, India NFHS 2005-06 (Continued)

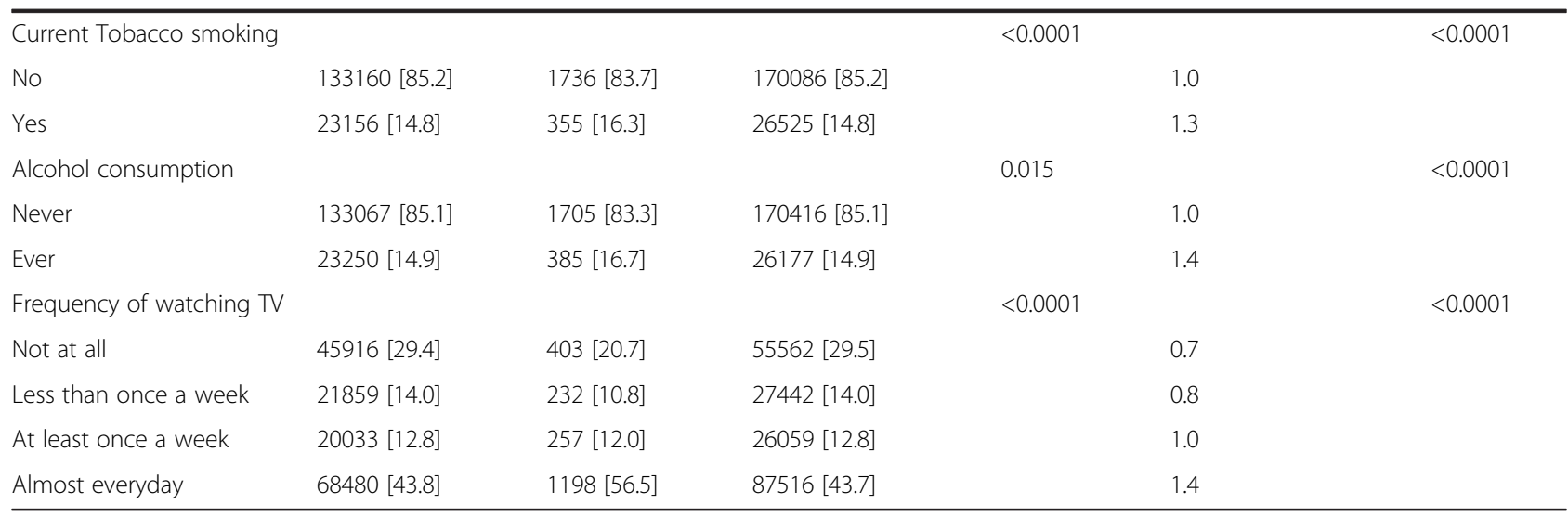

*Total participants varies slightly for individual variables depending on the number of missing values.

${ }^{a}$ Education: illiterate ( 0 years of education), literate but less than middle school complete (1-5 years of education), middle school complete (6-8 years of education), high school complete or more (9+ years of education).

${ }^{b}$ Scheduled castes and scheduled tribes are identified by the Government of India as socially and economically backward and needing protection from social injustice and exploitation. Other backward class is a diverse collection of intermediate castes that were considered low in the traditional caste hierarchy but are clearly above scheduled castes. Others is thus a default residual group that enjoys higher status in the caste hierarchy.

'Others include Buddhist, Jain, Jewish, Zoroastrian.

${ }^{d}$ The wealth index is based on following assets in the household: household electrification, type of windows, drinking water source, type of toilet facility, type of flooring, material of exterior walls, type of roofing, house ownership, ownership of a bank or post office account, and ownership of a mattress, a pressure cooker, a chair, a cot/bed, a table, an electric fan, a radio/transistor, a black and white television, a colour television, a sewing machine, a mobile telephone, any other telephone, a computer, a refrigerator, a watch or clock, a bicycle, a motorcycle or scooter, an animal-drawn cart, a car, a water pump, a thresher, and a tractor. eWomen who were pregnant at the time of the survey or women who had given birth during the two months preceding the survey were excluded from these measurements.

diet intake and diabetes prevalence in developing countries. Our finding suggest that persons consuming a lacto vegetarian, lacto-ovo vegetarian or semi-vegetarian diet had a lower likelihood of diabetes compared with those consuming non-vegetarian diet after adjustment for a number of socioeconomic and lifestyle factors. These findings may be explained by adverse effects of meat and fish, protective effects of typical constituents of lacto-vegetarian and lacto-ovo vegetarian diet which have been demonstrated elsewhere [23-26]. Our study indicates that the body mass index of Indian vegetarian diet consumers did not differ significantly from their non-vegetarian counterparts, but the male vegetarians appeared to be significantly $(\mathrm{p}<0.0001)$ uniformly leaner than female vegetarians. This association between vegetarianism and non-leanness is in line with a health study

Table 4 Unadjusted prevalence (\% with $\mathrm{Cl}$ ) of diabetes and obesity according to types of vegetarian diet consumption in adult Indian population $(n=156,317)$ aged $20-49$ years, NFHS 2005-06

\begin{tabular}{|c|c|c|c|c|c|c|c|}
\hline \multirow[t]{2}{*}{ Characteristics } & \multicolumn{6}{|c|}{ Type of diets } & \multirow{2}{*}{$\begin{array}{l}\text { P for trend } \\
\text { values* }\end{array}$} \\
\hline & Vegan & Lacto-vegetarian & Lacto-ovo vegetarian & Pesco-vegetarian & Semi-vegetarian & Non-vegetarian & \\
\hline Diabetes & $26[1.0]$ & $356[0.9]$ & $46[0.9]$ & $48[1.4]$ & $71[0.9]$ & $1223[1.2]$ & 0.002 \\
\hline $\mathrm{N}[\%], 95 \% \mathrm{Cl}$ & $0.6-1.7$ & $0.8-1.1$ & $0.6-1.3$ & $1.0-2.0$ & $0.7-1.1$ & $1.1-1.3$ & \\
\hline $\mathrm{BMI} \geq 23 \mathrm{~kg} / \mathrm{m}^{2}$ & 534 [21.5] & $9722[26.9]$ & $1163[24.9]$ & 650 [19.5] & $1690[21.8]$ & $21380[22.6]$ & $<0.001$ \\
\hline $\mathrm{N}[\%], 95 \% \mathrm{Cl}$ & $19.5-23.7$ & $26.3-27.5$ & $23.4-26.5$ & $17.8-21.3$ & $20.7-23.0$ & $22.3-23.0$ & \\
\hline $\mathrm{BMl} \geq 25 \mathrm{~kg} / \mathrm{m}^{2}$ & $286[11.5]$ & $5861[16.2]$ & $697[14.9]$ & $334[10.0]$ & $877[11.3]$ & $11996[12.7]$ & $<0.001$ \\
\hline $\mathrm{N}[\%], 95 \% \mathrm{Cl}$ & 10.0-13.2 & $15.7-16.7$ & $13.7-16.3$ & $8.0-11.3$ & $10.5-12.2$ & $12.4-13.0$ & \\
\hline $\mathrm{BMl} \geq 30 \mathrm{~kg} / \mathrm{m}^{2}$ & $58[2.3]$ & $1311[3.6]$ & $140[3.0]$ & $56[1.7]$ & $156[1.6]$ & $2269[2.4]$ & $<0.001$ \\
\hline $\mathrm{N}[\%], 95 \% \mathrm{Cl}$ & $1.7-3.2$ & $3.4-3.9$ & $2.5-3.7$ & $1.2-2.4$ & $1.2-2.4$ & $2.3-2.5$ & \\
\hline BMl, mean $[ \pm \mathrm{SD}]$ & $20.5[ \pm 4.2]$ & $21.2[ \pm 4.5]$ & $21.0[ \pm 4.1]$ & $20.3[ \pm 3.8]$ & $20.6[ \pm 4.0]$ & $20.7[ \pm 4.1]$ & \\
\hline
\end{tabular}

*P for trend values has been obtained from Likelihood ratio test for no difference between the groups for types of vegetarian diet ignoring the correlated data. As the non-vegetarian group was expected to have the highest and the rural group the lowest levels of diabetes and BMl, trend tests were carried out scoring the groups 1 to 5 and using likelihood ratio tests. 
Table 5 Percentage distribution of non-dietary variables according to types of vegetarian diet consumption in adult Indian population $(n=156,317)$ aged 20-49 years, NFHS 2005-06

\begin{tabular}{|c|c|c|c|c|c|c|c|}
\hline \multirow[t]{2}{*}{ Characteristics } & \multicolumn{6}{|c|}{ Type of diets } & \multirow[t]{2}{*}{ Chi sq $p$ values } \\
\hline & Vegan & $\begin{array}{l}\text { Lacto- } \\
\text { vegetarian }\end{array}$ & $\begin{array}{l}\text { Lacto-ovo } \\
\text { vegetarian }\end{array}$ & $\begin{array}{l}\text { Pesco- } \\
\text { vegetarian }\end{array}$ & $\begin{array}{l}\text { Semi- } \\
\text { vegetarian }\end{array}$ & $\begin{array}{l}\text { Non- } \\
\text { vegetarian }\end{array}$ & \\
\hline Age & & & & & & & $<0.001$ \\
\hline $20-29 y$ & $1097[1.7]$ & $14846[22.5]$ & $2535[3.8]$ & 1234 [1.9] & $3772[5.7]$ & $42553[64.4]$ & \\
\hline $30-39 y$ & $856[1.6]$ & $12758[24.3]$ & $1553[3.0]$ & $1203[2.3]$ & $2550[4.9]$ & 33648 [64.0] & \\
\hline $40-49 y$ & $609[1.6]$ & $10196[27.0]$ & $913[2.4]$ & $1005[2.7]$ & $1817[4.8]$ & 23172 [61.4] & \\
\hline Sex & & & & & & & $<0.001$ \\
\hline Men & $406[0.7]$ & $10683[18.8]$ & 2226 [3.9] & $955[1.7]$ & $3465[6.1]$ & 39009 [68.7] & \\
\hline Women & $2156[2.2]$ & $27118[27.2]$ & $2775[2.8]$ & $2487[2.5]$ & $4675[4.7]$ & 60364 [60.6] & \\
\hline Education & & & & & & & $<0.001$ \\
\hline No education & $1207[2.1]$ & $12896[22.7]$ & 1115 [2.0] & $1363[2.4]$ & $3183[5.6]$ & $36957[65.2]$ & \\
\hline Primary & $455[1.9]$ & $5645[20.6]$ & $599[2.4]$ & $628[2.6]$ & 1304 [5.3] & $16462[67.2]$ & \\
\hline Secondary & 755 [1.3] & $14505[24.8]$ & 2199 [3.8] & $1185[2.0]$ & $2967[5.1]$ & 36817 [63.0] & \\
\hline Higher and above & $125[0.8]$ & $5355[32.2]$ & $1087[6.5]$ & $265[1.6]$ & $686[4.1]$ & $9120[54.8]$ & \\
\hline Caste/tribe & & & & & & & $<0.001$ \\
\hline Scheduled caste & $456[1.6]$ & $4343[15.0]$ & $836[2.9]$ & $731[2.5]$ & 2018 [7.0] & 20545 [71.0] & \\
\hline Scheduled tribe & $311[2.4]$ & $1603[12.6]$ & $271[2.1]$ & $264[2.1]$ & $709[5.6]$ & $9576[75.2]$ & \\
\hline Other backward class & $1019[1.7]$ & $16614[27.2]$ & $2160[3.5]$ & $1319[2.2]$ & $3026[5.0]$ & $36839[60.4]$ & \\
\hline General & $761[1.6]$ & $15087[30.9]$ & $1692[3.5]$ & 1014 [2.1] & 2110 [4.3] & $28189[57.7]$ & \\
\hline Missing caste & $9[0.2]$ & $109[2.6]$ & $31[0.7]$ & $92[2.2]$ & $222[5.2]$ & 3808 [89.2] & \\
\hline Religion & & & & & & & $<0.001$ \\
\hline Hindu & 2358 [1.9] & $35337[27.7]$ & $4522[3.6]$ & $3069[2.4]$ & $6192[4.9]$ & 75897 [59.6] & \\
\hline Muslim & $78[0.4]$ & $272[1.4]$ & $196[1.0]$ & $260[1.3]$ & $1417[7.2]$ & 17558 [88.8] & \\
\hline Christian & $6[0.2]$ & $27[0.7]$ & $32[0.8]$ & $65[1.7]$ & $102[2.7]$ & 3585 [93.9] & \\
\hline Sikh & $75[2.6]$ & $1561[54.9]$ & $170[6.0]$ & $10[0.4]$ & $286[10.1]$ & $742[26.1]$ & \\
\hline Others & $45[1.8]$ & $602[24.1]$ & $81[3.2]$ & $38[1.5]$ & $143[5.7]$ & $1591[63.6]$ & \\
\hline Wealth quintiles & & & & & & & $<0.001$ \\
\hline Lowest & $586[2.2]$ & $4777[18.1]$ & $427[1.6]$ & $789[3.0]$ & $1051[4.0]$ & 18759 [71.1] & \\
\hline Second & $509[1.8]$ & $6225[21.7]$ & $735[2.6]$ & $741[2.6]$ & $1515[5.3]$ & $19026[66.2]$ & \\
\hline Middle & $468[1.5]$ & $6768[21.7]$ & $846[2.7]$ & $725[2.3]$ & 1997 [6.4] & 20428 [65.4] & \\
\hline Fourth & $516[1.5]$ & $7760[23.1]$ & $1167[3.5]$ & $667[2.0]$ & 1944 [5.8] & $21506[64.1]$ & \\
\hline Highest & $483[1.3]$ & $12270[33.7]$ & $1826[5.0]$ & $521[1.4]$ & $1633[4.5]$ & 19653 [54.0] & \\
\hline Place of residence & & & & & & & $<0.001$ \\
\hline Urban & $757[1.4]$ & $12685[23.4]$ & $2259[4.2]$ & 904 [1.7] & $2964[5.5]$ & 34565 [63.9] & \\
\hline Rural & $1804[1.8]$ & $25116[24.6]$ & $2741[2.7]$ & $2538[2.5]$ & $5176[5.1]$ & $64808[63.4]$ & \\
\hline Current Tobacco smoking & & & & & & & $<0.001$ \\
\hline No & $2388[1.8]$ & $34150[25.6]$ & $4258[3.2]$ & $3026[2.3]$ & $6687[5.0]$ & $82651[62.1]$ & \\
\hline Yes & $173[0.7]$ & $3651[15.8]$ & $742[3.2]$ & $416[1.8]$ & $1453[6.3]$ & $16721[72.2]$ & \\
\hline Alcohol consumption & & & & & & & $<0.001$ \\
\hline Never & $2496[1.9]$ & $36605[27.5]$ & $4320[3.2]$ & $3102[2.3]$ & $6707[5.0]$ & $79745[60.0]$ & \\
\hline Ever & $66[0.3]$ & $1196[5.1]$ & $681[2.9]$ & $339[1.5]$ & $1431[6.1]$ & $19611[84.1]$ & \\
\hline
\end{tabular}


Table 5 Percentage distribution of non-dietary variables according to types of vegetarian diet consumption in adult Indian population ( $n=156,317$ ) aged 20-49 years, NFHS 2005-06 (Continued)

\begin{tabular}{|c|c|c|c|c|c|c|c|}
\hline Frequency of watching $T$ & & & & & & & $<0.001$ \\
\hline Not at all & $1177[2.6]$ & $12046[26.2]$ & $937[2.0]$ & $1269[2.8]$ & $2170[4.7]$ & 28318 [61.7] & \\
\hline Less than once a week & $245[1.1]$ & $4789[21.9]$ & $588[2.7]$ & $467[2.1]$ & $884[4.0]$ & $14886[68.1]$ & \\
\hline At least once a week & $314[1.6]$ & $4639[23.2]$ & $663[3.3]$ & $476[2.4]$ & $1128[5.6]$ & $12814[64.0]$ & \\
\hline Almost everyday & $824[1.2]$ & $16322[23.8]$ & $2812[4.1]$ & $1230[1.8]$ & $3956[5.8]$ & $43336[63.3]$ & \\
\hline Total & $2560[1.6]$ & $37797[24.2]$ & $5002[3.2]$ & $3446[2.2]$ & 8140 [5.2] & 99372 [63.6] & 156317 \\
\hline
\end{tabular}

among the Barbados Seventh-Day Adventists which found self-reported vegetarians of less than 5 years did not differ significantly from the non-vegetarians [27].

Our results are in consistent with those of previous studies among various seventh-Day Adventist churchgoers $[1,2,5,6,28,29]$, several other studies conducted in western countries [10,30-37] and an Indian study [38] which showed that increased conformity to vegetarian diets is protected against risk of type 2 diabetes and hypertension. Findings from an accumulating number of studies have also shown evidence that most vegetarian diets are not only nutritionally adequate but also associated with lower risk of certain chronic diseases, when compared with the effects of a more typical western diets [36]. Evidence from a number of other observational studies shows that certain dietary constituents are associated with protection against diabetes through the pathway of insulin sensitivity which is also confirmed through food trials [10]. A reduced risk of chronic disease has been reported in populations of vegetarians living in affluent countries [39-41] and in case-control comparisons in developing countries [42]. Reduced consumption of animal fat and increased consumption of fruit, vegetables, foods that have a low glycemic index such as beans, legumes, nuts and cereals including whole grain and foods that reduce oxidative stress and chronic inflammation [2] may underlie such a protective effect. Whole grain is also a potential contributor to reduced diabetes risk in vegetarians and accumulating evidences from various prospective studies (both in men and women) and several meta-analyses shows that consumption of whole grains may reduce risk of chronic diseases including type 2 diabetes [43-49]. A recent meta-analysis [49] shows that the summary relative risk per 3 servings per day was $0.68\left(95 \% \mathrm{CI}: 0.58-0.81 ; \mathrm{I}^{2}=82 \% ; \mathrm{n}=10\right)$ for whole grains and 0.95 (95\%CI:0.88-1.04; $\left.\mathrm{I}^{2}=53 \% ; \mathrm{n}=6\right)$ for refined grains. In addition to the influence of fiber and glycemic load on postprandial glucose and insulin response [50], whole grains may also reduce the risk of type 2 diabetes through the action of nutrients such as vitamin $\mathrm{E}$ and magnesium [50-52].

We did not find an expected association between vegans (who eat no animal products) and significant reduced diabetes prevalence in our study contrary to other western studies. The term 'vegan' which may not be correctly asked/interpreted/self-reported in Indian context might be an important reason for this unexpected association, as because vegans in western countries necessarily do not have any form of dairy, including butter or ghee or any animal products, including honey which might not be the case for Indian vegans; selfreported vegans in India probably have butter/ghee/ honey in their diet. Second, it may be likely that vegans may be eating more of the refined-rice diets than nonvegetarians and unlike in the West, vegans in India would not be doing it for health-conscious/political reasons. Also, since this is a cross-sectional study, we may have some reverse casuality - diabetes patients changing to a vegan diet after the diabetes diagnosis. Another issue could be the statistical power. We found the odds ratio in vegan men is very similar to that in lactovegetarian men, while the result in women is null. Thus, the small sample size in the vegan group, which constituted only $1.6 \%$ of the sample, might have influenced the study results. However, though the more recent official statements from American Diabetes Association has clearly described vegetarian diets as healthful [53] and some studies $[1,2]$ shows that vegans have a least risk of type 2 diabetes, still the association between vegan diet and diabetes risk is open to question.

The notion that animal protein stimulates insulin secretion and possibly insulin resistance was proposed decades ago [54]. Red and processed meat consumption has been associated with increased risk of type 2 diabetes in a large number of cohort studies in the west [6,55-57]. Meat intake was associated with a higher risk of diagnosed diabetes in a study in Seventh-Day Adventists [8]. Several other studies around the globe have also reported an increased risk of diabetes or type 2 diabetes with a higher intake of processed meat [7,9,58-62], red meat $[7,58,59,62-64]$ and total meat $[7,9,65]$, but in some studies the results have been inconsistent $[55,61]$.

The categories that we have used to distinguish different types of diet in our study is internationally recognized $[2,8,17,18]$ and have also proven to be categories that have markedly different risks of common diseases 
Table 6 Multivariable logistic regression analysis (odds ratio with 95\% confidence interval) of the relation between types of vegetarian diet and self reported diabetes, in adult Indian population aged 20-49 years, NFHS-3 2005-06

\begin{tabular}{|c|c|c|c|c|c|c|}
\hline Characteristics & Unadjusted OR $[95 \% \mathrm{Cl}]$ & $P$ values & Adjusted* OR $[95 \% \mathrm{Cl}]$ & $P$ values & Adjusted $\psi$ OR $[95 \% \mathrm{Cl}]$ & $P$ values \\
\hline \multicolumn{7}{|c|}{ Types of vegetarian diet } \\
\hline Non-vegetarian (ref) & 1 & & 1 & & 1 & \\
\hline Semi-vegetarian & $0.71[0.56-0.90]$ & 0.005 & $0.77[0.60-0.98]$ & 0.034 & $0.76[0.59-0.96]$ & 0.024 \\
\hline Pesco-vegetarian & $1.13[0.84-1.51]$ & 0.426 & $1.15[0.85-1.54]$ & 0.365 & $1.09[0.81-1.46]$ & 0.589 \\
\hline Lacto-ovo vegetarian & $0.74[0.55-1.00]$ & 0.047 & $0.70[0.51-0.96]$ & 0.025 & $0.73[0.54-0.99]$ & 0.044 \\
\hline Lacto-vegetarian & $0.76[0.68-0.86]$ & $<0.001$ & $0.67[0.58-0.76]$ & $<0.001$ & $0.66[0.58-0.75]$ & $<0.001$ \\
\hline Vegan & $0.81[0.55-1.20]$ & 0.289 & $0.91[0.61-1.36]$ & 0.643 & 0.89 [0.59-1.32] & 0.553 \\
\hline \multicolumn{7}{|l|}{ Age } \\
\hline 20-29y (ref) & 1 & & 1 & & 1 & \\
\hline $30-39 y$ & $3.23[2.74-3.80]$ & $<0.001$ & 2.83 [2.39-3.35] & $<0.001$ & $3.32[2.82-3.91]$ & $<0.001$ \\
\hline $40-49 y$ & 9.20 [7.91-10.69] & $<0.001$ & $7.78[6.64-9.12]$ & $<0.001$ & 9.39 [8.05-10.95] & $<0.001$ \\
\hline \multicolumn{7}{|l|}{ Sex } \\
\hline Men (ref) & 1 & & 1 & & 1 & \\
\hline Women & $0.83[0.75-0.91]$ & $<0.001$ & $0.87[0.76-0.99]$ & 0.029 & $0.94[0.83-1.06]$ & 0.298 \\
\hline \multicolumn{7}{|l|}{ Education } \\
\hline No education (ref) & 1 & & 1 & & 1 & \\
\hline Primary & 1.46 [1.27-1.69] & $<0.001$ & 1.20 [1.03-1.40] & 0.022 & $1.23[1.060-1.44]$ & 0.008 \\
\hline Secondary & $1.53[1.36-1.71]$ & $<0.001$ & $1.22[1.05-1.41]$ & 0.008 & 1.28 [1.11-1.47] & 0.001 \\
\hline Higher and above & 1.63 [1.39-1.91] & $<0.001$ & $1.05[0.85-1.28]$ & 0.672 & 1.11 [0.91-1.36] & 0.300 \\
\hline \multicolumn{7}{|l|}{ Caste/tribe } \\
\hline Scheduled caste (ref) & 1 & & 1 & & 1 & \\
\hline Scheduled tribe & $0.40[0.30-0.54]$ & $<0.001$ & $0.47[0.34-0.63]$ & $<0.001$ & $0.47[0.35-0.63]$ & $<0.001$ \\
\hline Other backward class & $0.98[0.85-1.12]$ & 0.714 & 0.84 [0.73-0.98] & 0.022 & 0.86 [0.75-0.99] & 0.039 \\
\hline General & 1.38 [1.21-1.58] & $<0.001$ & $0.95[0.81-1.10]$ & 0.459 & 0.98 [0.84-1.13] & 0.751 \\
\hline Missing caste & 1.61 [1.26-2.06] & $<0.001$ & 1.18 [0.90-1.55] & 0.244 & 1.20 [0.91-1.59] & 0.199 \\
\hline \multicolumn{7}{|l|}{ Religion } \\
\hline Hindu (ref) & 1 & & 1 & & 1 & \\
\hline Muslim & 1.26 [1.11-1.44] & $<0.001$ & 1.14 [0.98-1.33] & 0.100 & 1.16 [0.99-1.35] & 0.062 \\
\hline Christian & 1.85 [1.46-2.33] & $<0.001$ & 1.31 [1.02-1.68] & 0.035 & 1.36 [1.07-1.74] & 0.014 \\
\hline Sikh & 1.25 [0.91-1.73] & 0.173 & 0.85 [0.61-1.19] & 0.333 & $0.96[0.69-1.33]$ & 0.787 \\
\hline Others & $0.83[0.54-1.26]$ & 0.375 & $0.70[0.44-1.10]$ & 0.119 & $0.74[0.48-1.13]$ & 0.159 \\
\hline \multicolumn{7}{|l|}{ Wealth quintiles } \\
\hline Lowest (ref) & 1 & & 1 & & 1 & \\
\hline Second & 1.56 [1.27-1.92] & $<0.001$ & 1.40 [1.13-1.73] & 0.002 & $1.42[1.15-1.76]$ & 0.001 \\
\hline Middle & $1.38[1.12-1.71]$ & 0.002 & 1.05 [0.84-1.32] & 0.654 & 1.16 [0.93-1.44] & 0.201 \\
\hline Fourth & 2.39 [1.98-2.90] & $<0.001$ & $1.53[1.22-1.93]$ & $<0.001$ & $1.80[1.44-2.25]$ & $<0.001$ \\
\hline Highest & $3.77[3.15-4.51]$ & $<0.001$ & $1.87[1.45-2.41]$ & $<0.001$ & $2.50[1.96-3.19]$ & $<0.001$ \\
\hline \multicolumn{7}{|l|}{ Place of residence } \\
\hline Urban & $1.96[1.79-2.16]$ & $<0.001$ & 1.20 [1.07-1.35] & 0.002 & 1.24 [1.10-1.39] & $<0.001$ \\
\hline Rural (ref) & 1 & & 1 & & 1 & \\
\hline \multicolumn{7}{|c|}{ Body Mass Index $\left(\mathrm{kg} / \mathrm{m}^{2}\right)$} \\
\hline$\leq 18.5 \mathrm{~kg} / \mathrm{m}^{2}$ & $0.56[0.49-0.64]$ & $<0.001$ & $0.75[0.65-0.88]$ & $<0.001$ & - & \\
\hline $18.5-22.9 \mathrm{~kg} / \mathrm{m}^{2}$ (ref) & 1 & & 1 & & & \\
\hline
\end{tabular}


Table 6 Multivariable logistic regression analysis (odds ratio with 95\% confidence interval) of the relation between types of vegetarian diet and self reported diabetes, in adult Indian population aged 20-49 years, NFHS-3 2005-06 (Continued)

\begin{tabular}{|c|c|c|c|c|c|c|}
\hline $23.0-24.9 \mathrm{~kg} / \mathrm{m}^{2}$ & $2.57[2.26-2.93]$ & $<0.001$ & $1.64[1.42-1.91]$ & $<0.001$ & - & \\
\hline$\geq 25.0 \mathrm{~kg} / \mathrm{m}^{2}$ & $4.06[3.65-4.52]$ & $<0.001$ & $2.21[1.95-2.51]$ & $<0.001$ & - & \\
\hline \multicolumn{7}{|l|}{ Current tobacco smoking } \\
\hline No (ref) & 1 & & 1 & & 1 & \\
\hline Yes & $1.13[0.99-1.28]$ & 0.069 & $0.98[0.84-1.15]$ & 0.803 & $0.93[0.80-1.09]$ & 0.379 \\
\hline \multicolumn{7}{|l|}{ Alcohol consumption } \\
\hline Never (ref) & 1 & & 1 & & 1 & \\
\hline Ever & $1.15[1.01-1.30]$ & 0.029 & 1.01 [0.87-1.19] & 0.870 & $1.05[0.90-1.23]$ & 0.519 \\
\hline \multicolumn{7}{|c|}{ Frequency of watching TV } \\
\hline Not at all (ref) & 1 & & 1 & & 1 & \\
\hline Less than once a week & $1.10[0.92-1.31]$ & 0.307 & $0.89[0.74-1.07]$ & 0.202 & $0.92[0.76-1.10]$ & 0.345 \\
\hline At least once a week & 1.34 [1.13-1.58] & 0.001 & $0.95[0.79-1.14]$ & 0.594 & $1.00[0.84-1.20]$ & 0.975 \\
\hline Almost everyday & 1.84 [1.63-2.08] & $<0.001$ & $0.91[0.78-1.07]$ & 0.258 & 1.04 [0.89-2.21] & 0.652 \\
\hline
\end{tabular}

${ }^{*}$ Adjusted for all factors; $\Psi$ Adjusted for all factors except BMl; OR- indicates odds ratios; ref- indicates reference category.

such as diabetes and hypertension in cohort $[1,2,66]$ and observational studies in UK [37] but it is possible that the more-refined categories may provide better comparability.

The strengths of our study include the use of large nationally representative study sample which allows comparisons to be made between men and women and the ability to examine this association in adult Indian population. Also rigorous efforts were made in the NFHS-3 to obtain reliable self-reported data: the survey used local terminology and commonly understood terms to describe the disease, rigorously trained interviewers, supervisors and standard quality checks [16] (also see www.dhsprogram.com).

The prevalence of diabetes in this large nationally representative survey was comparatively low (1.1\%) than studies conducted in selected geographical areas or cities in India [11,14,67-71]. The low diabetes prevalence in our study reflects the young age of this population, the use of self-reports rather than biochemical assessments and sampling from the general population that included a high proportion of respondents in rural areas [72]. Our study has added to this literature using a national population health survey with good coverage in rural areas across India. Estimates from a recent study of rural-urban migrants showed an age-adjusted prevalence of diabetes (diagnosed using both self-reports and fasting blood glucose in relatively affluent populations) of $10-15 \%$ in urban people and $5-6 \%$ in rural people of similar age to those recruited in NFHS-3 [73]. In most urban parts of India the health system is well enough developed for diagnosis of symptomatic diabetes, but at younger ages $(<30$ years) diabetes may not be symptomatic and NFHS-3 prevalence estimates are undoubtedly conservative, particularly for rural India where diagnosis may be much less likely to occur [74]. However, this ascertainment bias is unlikely to have been differential with respect to types of vegetarian diet consumption. In other words, although we clearly have sub-set of disease, it's unlikely to be systematically different from entire group in terms of dietary patterns.

Previous research has shown a good agreement for self-reported diabetes when compared with medical records in a US population [75] and that self-reported health conditions demonstrate the expected relationship with socioeconomic status in India [76]. Studies in India also have shown that the difference between self reports and objective measurements according to education and awareness levels does not preclude the use of selfreports $[72,76]$. On the contrary to the prevailing view that there is a positive (or null) association between measures of socio economic status and self-reported poor health/morbidities in less-developed countries, and that any potential "under-reporting" is not only smaller than the difference in prevalence of illness between the socially disadvantaged and the advantaged, the study by Subramanian et al. [76] showed that the same is even true within groups with the same objective diagnosis. In addition, our analyses considering respondents who reported 'unknown' for diabetes status were nearly identical to the main analyses (data not shown). Although our sample was relatively young $(<50$ years for women and men both), it is representative of the young population profile of India; 84\% of the Indian adult population (1869 years) and $47 \%$ of the total Indian population at all ages fall within the ages covered by this study [77]. Our study does exclude approximately $14 \%$ of the Indian population (men and women over age 50) due to the sample design of the NFHS. The prevalence of diabetes 
Table 7 Multivariable logistic regression analysis (odds ratio with 95\% confidence interval) of the relation between types of vegetarian diet and self reported diabetes, in men $(n=56742)$ and women $(n=99574)$ aged 20-49 years, NFHS-3 2005-06

\begin{tabular}{|c|c|c|c|c|c|c|}
\hline Predictors & Unadjusted OR [95\% Cl] & $P$ values & Adjusted* OR [95\% Cl] & $P$ values & Adjusted $\Psi$ OR $[95 \% \mathrm{Cl}]$ & $P$ values \\
\hline \multicolumn{7}{|l|}{ Men } \\
\hline \multicolumn{7}{|l|}{ Types of vegetarian diet } \\
\hline Non-vegetarian (ref) & 1 & & 1 & & 1 & \\
\hline Semi-vegetarian & $0.47[0.31-0.72]$ & $<0.001$ & $0.45[0.29-0.71]$ & 0.001 & $0.48[0.32-0.73]$ & 0.001 \\
\hline Pesco-vegetarian & $0.78[0.42-1.44]$ & 0.426 & $0.80[0.43-1.50]$ & 0.488 & $0.77[0.41-1.43]$ & 0.407 \\
\hline Lacto-ovo vegetarian & $0.74[0.49-1.13]$ & 0.165 & $0.63[0.39-1.00]$ & 0.050 & $0.72[0.47-1.11]$ & 0.134 \\
\hline Lacto-vegetarian & $0.79[0.64-0.96]$ & 0.020 & $0.66[0.52-0.82]$ & $<0.001$ & $0.65[0.52-0.81]$ & $<0.001$ \\
\hline Vegan & $0.67[0.24-1.85]$ & 0.438 & $0.70[0.25-1.96]$ & 0.498 & $0.66[0.24-1.83]$ & 0.424 \\
\hline \multicolumn{7}{|c|}{ Body Mass Index $\left(\mathrm{kg} / \mathrm{m}^{2}\right)$} \\
\hline$\leq 18.5 \mathrm{~kg} / \mathrm{m}^{2}$ & $0.75[0.60-0.93]$ & 0.009 & $0.85[0.68-1.06]$ & 0.147 & - & \\
\hline $18.5-22.9 \mathrm{~kg} / \mathrm{m}^{2}(\mathrm{ref})$ & 1 & & 1 & & - & \\
\hline $23.0-24.9 \mathrm{~kg} / \mathrm{m}^{2}$ & $2.29[1.85-2.83]$ & $<0.001$ & $1.63[1.30-2.03]$ & $<0.001$ & - & \\
\hline$\geq 25.0 \mathrm{~kg} / \mathrm{m}^{2}$ & $3.06[2.53-3.71]$ & $<0.001$ & $1.80[1.46-2.23]$ & $<0.001$ & - & \\
\hline \multicolumn{7}{|l|}{ Women } \\
\hline \multicolumn{7}{|l|}{ Types of vegetarian diet } \\
\hline Non-vegetarian (ref) & 1 & & 1 & & 1 & \\
\hline Semi-vegetarian & $0.92[0.68-1.23]$ & 0.561 & 1.09 [0.81-1.47] & 0.582 & 1.03 [0.77-1.39] & 0.842 \\
\hline Pesco-vegetarian & 1.33 [0.96-1.86] & 0.090 & 1.33 [0.95-1.87] & 0.101 & $1.24[0.88-1.74]$ & 0.220 \\
\hline Lacto-ovo vegetarian & $0.72[0.47-1.10]$ & 0.128 & $0.77[0.50-1.19]$ & 0.238 & $0.75[0.49-1.14]$ & 0.176 \\
\hline Lacto-vegetarian & $0.78[0.67-0.90]$ & 0.001 & $0.70[0.59-0.82]$ & $<0.001$ & $0.69[0.59-0.81]$ & $<0.001$ \\
\hline Vegan & $0.89[0.58-1.37]$ & 0.606 & $1.01[0.65-1.56]$ & 0.984 & $0.98[0.64-1.52]$ & 0.931 \\
\hline \multicolumn{7}{|c|}{ Body Mass Index $\left(\mathrm{kg} / \mathrm{m}^{2}\right)$} \\
\hline$\leq 18.5 \mathrm{~kg} / \mathrm{m}^{2}$ & $0.60[0.49-0.74]$ & $<0.001$ & $0.68[0.56-0.84]$ & $<0.001$ & - & \\
\hline $18.5-22.9 \mathrm{~kg} / \mathrm{m}^{2}(\mathrm{ref})$ & 1 & & 1 & & - & \\
\hline $23.0-24.9 \mathrm{~kg} / \mathrm{m}^{2}$ & $2.23[1.83-2.71]$ & $<0.001$ & $1.63[1.34-2.00]$ & $<0.001$ & - & \\
\hline$\geq 25.0 \mathrm{~kg} / \mathrm{m}^{2}$ & $4.32[3.72-5.01]$ & $<0.001$ & $2.44[2.07-2.87]$ & $<0.001$ & - & \\
\hline
\end{tabular}

*Adjusted for age, education, caste/tribe, religion, wealth quintiles, place of residence, BMI, current tobacco smoking, alcohol consumption and frequency of watching TV. $\Psi$ Adjusted for all factors except BMI. OR: odds ratios. ref- reference category.

increases with age and whether a similar socioeconomic status-diabetes relationship exists among middle and older age groups in all parts India is not clear [72], although our findings are consistent with the previous studies that have included older ages.

The current national estimate for diabetes prevalence in India is about $7 \%$ of the adult population aged $20-79$ years [72]; estimates being based on three relatively recent and large scale studies using a combination of oral glucose tolerance testing and self-reports of diabetes $[11,78]$. There continues to be considerable uncertainty in estimates of diabetes for the whole of India due to the limited study locations (with a focus on urban areas), wide variation in survey sampling methodology, differences in diabetes diagnostic criteria and age groups studied [72]. These differences in study design have hindered direct comparison of the prevalence between studies, across regions and over time. The NFHS-3 provides an important benchmark because it is the first nationally representative survey of diabetes in India. Even if the prevalence estimates of diabetes have been underestimated in the NFHS-3, the observed diet-diabetes associations are reasonable and significant, and can be comparable to cohort and prospective studies on similar association in the west. Previous studies have largely overlooked the importance of modifiable dietary factors, which may be a key determinant of diabetes in Indians, given the varied nature of Indian diets. Further large-scale population-based surveys can be strengthened by using simple finger-prick blood glucose measurements in addition to self-reports.

In our analyses, the cross-sectional design precludes causal inferences and we were limited to the questions 
used to elicit lifestyle and dietary information. Given the high proportion of undiagnosed diabetes in developing countries including India (www.worlddiabetesfoundation.org) where less than half of people with diabetes are diagnosed, there is a possibility that the exposure was associated with the likelihood of testing for diabetes, which may result in detection bias. Importantly the entire study may be with known diabetic subjects who would have altered diet and hence might have increased or decreased vegetarian diet consumption due to the dietary advice based on diabetes control and complications of diabetes like nephropathy. General dietary advice given to diabetic subjects is to include more whole grains, legumes, fruits and green leafy and other vegetables as this is evident in our data where more than $90 \%$ of the self-reported diabetics did report 'daily' or 'weekly' consumption of legumes, vegetables and fruits-all suggest that the dietary choices of self-reported diabetic subjects might have been modified to manage diabetes. However, despite these shortcomings rigorous precautions were taken in the NFHS to obtain reliable self-reported data such as the survey used the local terminology and commonly understood term of the disease, rigorously trained interviewers and supervisors and standard quality checks.

Nevertheless, our study has some other limitations. Misclassification of dietary information, although unavoidable, would most likely be non-differential and thus may attenuate the true association. There were relatively small numbers in some of the dietary categories, which should be considered when interpreting the findings in relation to these diets. There might be limitation of the dietary assessment method in NFHS-3 as well since there may be other foods that are associated with diabetes that are not asked to the respondents. We were also unable to distinguish between Type 1 and 2 diabetes diagnoses. Since the NFHS-3 questionnaire is interviewer administered, information on the inter rater compatibility, reproducibility and validity of questionnaire would be critical to evaluate the ability of such questionnaire to measure true dietary intake. But NFHS-3 being a part of Demographic and Health Surveys (available at www.dhsprogram.com) which is conducted in more than 80 countries with similar questionnaire seems to be fairly valid to get an overall picture of frequency of dietary intake in a population [79]. However, under- and over-reporting could lead to a biased estimation of the association between dietary factors and diabetes. Although we adjusted for several confounding variables, we cannot exclude the possibility of residual confounding. However, if this was the case, similar effects would be expected for dietary components that are related to greater affluence, which was not observed.

Another limitation of our study is reliance on selfreports of diabetes which has resulted in a marked underestimation of prevalence, and its focus on people $<60$ years in whom diabetes is less common [74]. Selfreported data, especially in rural areas, can be flawed owing to several factors such as lack of awareness, low educational status, limited access to health services and hesitation to disclose diagnosed diseases. But in developing countries, self-reporting should not be a prohibitive limitation as medically diagnosed and/or biomarkerconfirmed prevalence estimates are nearly impossible for nationwide prevalence estimates in low- resource and low-access settings such as India. Also since, the low and middle income countries has only very limited nutrition and health outcomes data, NFHS-3 is therefore the best available dataset to examine the relationship.

Valid data on physical activity were not available in NFHS-3 which is a limitation of this study since persons with healthier diets may be physically more active than other persons [80]. Therefore the lack of physical activity data may have confounded the results. Moreover, assessment of sedentary habits in this study was based on hours of TV watching. However, physical activity has in part been accounted for, indirectly, by adjusting for body mass index. In the present study, adjustment for socioeconomic and demographic factors, residential location, religion and caste/tribe status of the respondents did not markedly modify the adjusted result, suggesting that the associations are not completely explained by non-dietary lifestyle factors. Further studies are needed to determine whether the association between diet and diabetes is mediated by assumed nutrients or by lifestyle and socioeconomic and demographic factors related to frequency of food consumption.

The rising burden of diabetes in India requires a rapid response that integrates policies and programmes which enable effective prevention and control across diverse geographical and low-resource settings. Our findings on inverse association between types of vegetarian diet consumption and diabetes prevalence can be considered by policy-makers to promote healthy vegetarian diet consumption in Indian population and to discourage unhealthy non-vegetarian diets. There is, therefore, an opportunity to modify the direction and dimensions of this national epidemic through policy interventions (at the state level), which promote the availability, affordability and acceptability of vegetarian diets more specifically lacto vegetarian and lacto-ovo vegetarian diets and restrain the marketing and consumption of unhealthy non-vegetarian foods. This requires coordinated action at the level of governments, civil society and responsible sections of the food industry.

\section{Conclusions}

In conclusion, our findings are important for public health interventions in diabetes care in India which shows that, 
in a large sample of adult men and women in India, variants of vegetarian diets such as lacto-vegetarian and lacto-ovo vegetarian were associated with at least a 30\% lower risk of diabetes. These results add to the limited evidence in developing countries that shows potential benefits of consuming vegetarian diets to reduce the development of diabetes. These findings need further validation by longitudinal and clinical studies but may well have public health significance for the Indian population. These findings, if replicated using objective and comprehensive methods of dietary intake and diabetes, may inform the development of interventions to address the growing burden of overweight and diabetes in India.

\section{Endnotes}

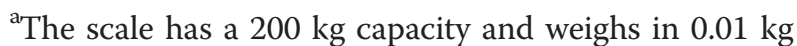
increments. The scale is powered by six AA batteries and has an "ON-OFF" switch located at the side of the scale. The SECA 874 digital floor scale is manufactured by Seca gmbh \& co. kg. Hammer Steindamm $9-25$. 22089 Hamburg. Germany. The scale can be procured directly from Seca. These instructions were adapted from instructions that accompany the scale and revised by Irwin J. Shorr, MPH, MPS.

'The state of a person's health in terms of the nutrients in his or her diet. In Indian context, it also means inadequate and poor diet and repeated exposure to disease and illness, may or may not be based on any clinical test or measurement.

\section{Competing interests}

The authors declare that they have no competing interests.

\section{Authors' contributions}

SA conceived the article. SA conducted and CM and SE supervised the statistical analysis. SA wrote the paper and CM, PD, SV and SE revised it for important intellectual content. SA is the guarantors of this work and, as such, had full access to all the data in the study and take responsibility for the integrity of the data and the accuracy of the data analysis. All authors gave final approval.

\section{Acknowledgements \\ An earlier version of the paper was accepted as a poster at the IUNS $20^{\text {th }}$ International Congress of Nutrition, Granada (Spain), September 15-20, 2013. SA is supported by a Wellcome Trust Strategic Award Grant No WT084674. The data for this research were collected by The Demographic and Health Surveys Program (www.dhsprogram.com), under a contract from the U.S. Agency for International Development. The authors acknowledge the support of International Institute for Population Sciences and Macro International for providing access to the 2005-06 Indian National Family Health Survey data. The authors are also thankful to the editor and the reviewers for their immensely helpful comments and suggestions and edits on the earlier draft of this paper.}

\section{Author details}

'South Asia Network for Chronic Disease, Public Health Foundation of India, Fourth Floor, Plot no 47, Sector 44, Gurgaon (Haryana)-122002, New Delhi, India. ${ }^{2}$ Department of Primary Care and Public Health, School of Public Health, Imperial College, London, UK. ${ }^{3}$ Department of Society, Human Development and Health, Harvard School of Public Health, Harvard University, Boston, USA. ${ }^{4}$ Department of Non-communicable Disease Epidemiology, London School of Hygiene and Tropical Medicine, London, UK.
Received: 9 April 2014 Accepted: 28 August 2014

Published: 5 September 2014

\section{References}

1. Tonstad S, Stewart K, Oda K, Batech M, Herring RP, Fraser GE: Vegetarian diets and incidence of diabetes in the Adventist health study-2. Nutr Metab Cardiovasc Dis 2013, 23(4):292-299.

2. Tonstad S, Butler T, Yan R, Fraser GE: Type of vegetarian diet, body weight, and prevalence of type 2 diabetes. Diabetes Care 2009, 32:791-796.

3. Rosell M, Appleby P, Spencer E, Key T: Weight gain over 5 years in 21,966 meat eating, fish-eating, vegetarian, and vegan men and women in EPIC-Oxford. Int J Obes 2006, 30:1389-1396.

4. Phillips F, Hackett A, Billington D, Stratton G: Effects of changing from a mixed to self-selected vegetarian diet on anthropometric measurements in UK adults. J Hum Nutr Diet 2004, 17:249-255.

5. Fraser GE: Associations between diet and cancer, ischemic heart disease, and all-cause mortality in non-Hispanic white California Seventh-day Adventists. Am J Clin Nutr 1999, 70(Suppl):532S-538S.

6. Appleby PN, Thorogood M, Mann J, Key TJ: Low body mass index in non-meat eaters: the possible roles of animal fat, dietary fibre and alcohol. Int J Obes Relat Metab Disord 1998, 22:454-460.

7. Fung $T$, Schulze M, Manson JE, Willett WC, Hu FB: Dietary patterns, meat intake, and the risk of type 2 diabetes in women. Arch Intern Med 2004, 164:2235-2240.

8. Snowdon DA, Phillips RL: Does a vegetarian diet reduce the occurrence of diabetes? Am J Public Health 1985, 75:507-512.

9. Vang A, Singh PN, Lee JW, Haddad EH, Brinegar CH: Meats, processed meats, obesity, weight gain and occurrence of diabetes among adults: findings from Adventist health studies. Ann Nutr Metab 2008, 52:96-104.

10. Jenkins DJA, Kendall CWC, Marchie A, Jenkins AL, Augustin LSA, Ludwig DS, Barnard ND, Anderson JW: Type 2 diabetes and the vegetarian diet. Am J Clin Nutr 2003, 78(Suppl):610S-616S.

11. Ramachandran A, Snehalatha C, Kapur A, Vijay V, Mohan V, Das AK, Rao PV, Yajnik CS, Prasanna Kumar KM, Nair JD, Diabetes Epidemiology Study Group in India (DESI): Diabetes Epidemiology Study Group in India (DESI): high prevalence of diabetes and impaired glucose tolerance in India: national urban diabetes survey. Diabetologia 2001, 44:1094-1101.

12. Wild S, Roglic G, Green A, Sicree R, King H: Global prevalence of diabetes: estimates for the year 2000 and projections for 2030. Diabetes Care 2004, 27:1047-1053.

13. Yoon $\mathrm{KH}$, Jin $\mathrm{HL}$, Ji-Won $\mathrm{K}$, Cho JH, Choi YH, Ko SH, Zimmet P, Son HY: Epidemic obesity and type 2 diabetes in Asia. Lancet 2006, 368:1681-1688.

14. Ramachandran A, Snehalatha C: Current scenario of diabetes in India. $J$ Diabetes 2009, 1:18-28.

15. Diamond JED: Diabetes in India. Nature 2011,469:479

16. International Institute for Population Sciences, Macro International: National Family Health Survey (NFHS-3), 2005-06: India, Volume I. Mumbai: IIPS; 2007.

17. Key TJ, Davey GK, Appleby PN: Health benefits of a vegetarian diet. Proc Nutr Soc 1999, 58(2):271-275.

18. Famodu AA, Osilesi $\mathrm{O}$, Makinde $\mathrm{YO}$, Osonuga OA: Blood pressure and blood lipid levels among vegetarian, semi-vegetarian, and nonvegetarian Native Africans. Clin Biochem 1998, 31(7):545-549.

19. ICF International: MEASURE DHS Biomarker Field Manual. Calverton, Maryland, U.S.A: ICF International; 2012

20. Indian Consensus Group: Indian consensus for prevention of hypertension and coronary heart disease: a joint scientific statement of Indian society of hypertension and international college of nutrition. J Nutr Environ Med 1996, 6:309-318.

21. WHO expert consultation: Appropriate body-mass index for Asian populations and its implications for policy and intervention strategies. Lancet 2004, 363(9403):157-163.

22. Misra A, Chowbey P, Makkar BM, Vikram NK, Wasir JS, Chadha D, Joshi SR, Sadikot S, Gupta R, Gulati S, Munjal YP, Concensus Group: Consensus statement for diagnosis of obesity, abdominal obesity and the metabolic syndrome for Asian Indians and recommendations for physical activity, medical and surgical management. J Assoc Physicians India 2009, 57:163-170.

23. Liu S, Choi HK, Ford E, Song Y, Klevak A, Buring JE, Manson JE: A prospective study of dairy intake and the risk of type 2 diabetes in women. Diabetes Care 2006, 29(7):1579-1584. 
24. Tremblay A, Gilbert JA: Milk products, insulin resistance syndrome and type 2 diabetes. J Am Coll Nutr 2009, 28(Suppl 1):91S-102S.

25. Djoussé L, Kamineni A, Nelson TL, Carnethon M, Mozaffarian D, Siscovick D, Mukamal KJ: Egg consumption and risk of type 2 diabetes in older adults. Am J Clin Nutr 2010, 92(2):422-427.

26. Shin JY, Xun P, Nakamura Y, He K: Egg consumption in relation to risk of cardiovascular disease and diabetes: a systematic review and metaanalysis. Am J Clin Nutr 2013, 98:146-59.

27. Brathwaite N, Fraser HS, Modeste N, Broome H, King R: Obesity, diabetes, hypertension, and vegetarian status among Seventh-Day Adventists in Barbados: preliminary results. Ethn Dis 2003, 13(1):34-39.

28. Singh PN, Fraser GE, Knutsen SF, Lindsted DS, Bennett H: Validity of a physical activity questionnaire among African-American Seventh-day Adventists. Med Sci Sports Exer 2000, 33:468-475.

29. Butler TL, Fraser GE, Beeson WL, Knutsen SF, Herring RP, Chan J, Sabaté J, Montgomery S, Haddad E, Preston-Martin S, Bennett H, Jaceldo-Siegl K: Cohort profile: the Adventist Health Study-2 (AHS-2). Int J Epidemiol 2008, 37:260-265.

30. Dwyer JT: Health aspects of vegetarian diets. Am J Clin Nutr 1988, 48(3 Suppl):712-738

31. Montonen $\mathrm{J1}$, Knekt $\mathrm{P}$, Järvinen $\mathrm{R}$, Reunanen $\mathrm{A}$ : Dietary antioxidant intake and risk of type 2 diabetes. Diabetes Care 2004, 27(2):362-366.

32. Bedford JL, Barr SI: Diets and selected lifestyle practices of self-defined adult vegetarians from a population-based sample suggest they are more 'health conscious'. Int I Behav Nutr Phys Act 2005, 2:4.

33. Key TJ, Appleby PN, Rosell MS: Health effects of vegetarian and vegan diets. Proc Nutr Soc 2006, 65(1):35-41.

34. Barnard ND, Cohen J, Jenkins DJ, Turner-McGrievy G, Gloede L, Jaster B, Seidl K, Green AA, Talpers S: A low-fat vegan diet improves glycemic control and cardiovascular risk factors in a randomized clinical trial in individuals with type 2 diabetes. Diabetes Care 2006, 29(8):1777-1783.

35. Ginter E: Vegetarian diets, chronic diseases and longevity. Bratisl Lek Listy 2008, 109(10):463-466.

36. Fraser GE: Vegetarian diets: what do we know of their effects on some chronic diseases? Am J Clin Nutr 2009, 89:1607S-1612S.

37. Rimm EB, Ascherio A, Giovannucci E, Spiegelman D, Stampfer MJ, Willett WC: Vegetable, fruit, and cereal fiber intake and risk of coronary heart disease among men. J Am Med Assoc 1996, 275:447.

38. Zaman GS, Zaman FA, Arifullah M: Comparative risk of type 2 diabetes mellitus among vegetarians and non-vegetarians. Indian J Community Med 2010, 35(3):441-442

39. Orlich MJ, Singh PN, Sabaté J, Jaceldo-Siegl K, Fan J, Knutsen S, Beeson WL, Fraser GE: Vegetarian dietary patterns and mortality in Adventist health study 2. JAMA Intern Med 2013, 173(13):1230-1238.

40. Tantamango-Bartley Y, Jaceldo-Siegl K, Fan J, Fraser G: Vegetarian diets and he incidence of cancer in a low-risk population. Cancer Epidemiol Biomarkers Prev 2013, 22(2):286-294

41. Willett WC: Convergence of philosophy and science: the third international congress on vegetarian nutrition. Am J Clin Nutr 1999, 70(Suppl):434S-438S

42. Pais P, Pogue J, Gerstein H, Zachariah E, Savitha D, Jayprakash S, Nayak PR, Yusuf S: Risk factors for acute myocardial infarction in Indians: a casecontrol study. Lancet 1996, 348:358-363.

43. Liu S, Manson JE, Stampfer MJ, Hu FB, Giovannucci E, Colditz GA, Hennekens $\mathrm{CH}$, Willett WC: A prospective study of wholegrain intake and risk of type 2 diabetes mellitus in US women. Am J Public Health 2000, 90:1409-1415.

44. Meyer KA, Kushi LH, Jacobs DR Jr, Slavin J, Sellers TA, Folsom AR: Carbohydrates, dietary fiber, and incident type 2 diabetes in older women. Am J Clin Nutr 2000, 71:921-930.

45. Fung TT, Hu FB, Pereira MA, Liu S, Stampfer MJ, Colditz GA, Willett WC: Whole-grain intake and the risk of type 2 diabetes: a prospective study in men. Am J Clin Nutr 2002, 76:535-540.

46. Montonen J, Knekt P, Jarvinen R, Aromaa A, Reunanen A: Whole grain and fiber intake and the incidence of type 2 diabetes. Am J Clin Nutr 2003, 77:622-629.

47. Van Dam RM, Hu FB, Rosenberg L, Krishnan S, Palmer JR: Dietary calcium and magnesium, major food sources, and risk of type 2 diabetes in U.S. black women. Diabetes Care 2006, 29:2238-2243.

48. Ye EQ, Chacko SA, Chou EL, Kugizaki M, Liu S: Greater whole-grain intake is associated with lower risk of type 2 diabetes, cardiovascular disease, and weight gain. J Nutr 2012, 142(7):1304-1313.
49. Aune $D$, Norat $T$, Romundstad $P$, Vatten $L$ : Whole grain and refined grain consumption and the risk of type 2 diabetes: a systematic review and dose-response meta-analysis of cohort studies. Eur J Epidemiol 2013, 28(11):845-858. Doi: 10.1007/s10654-013-9852-5. Epub 2013 Oct 25.

50. Slavin JL, Martini MC, Jacobs DRJ, Marquart L: Plausible mechanisms for the protectiveness of whole grains. Am J Clin Nutr 1999, 70(suppl):459S-463S.

51. Paolisso G, D'Amore A, Giugliano D, Ceriello A, Varricchio M, D'Onofrio F: Pharmacologic doses of vitamin $E$ improve insulin action in healthy subjects and non-insulin-dependent diabetic patients. Am J Clin Nutr 1993, 57:650-656.

52. Salonen JT, Nyyssönen K, Tuomainen TP, Mäenpää PH, Korpela H, Kaplan GA, Lynch J, Helmrich SP, Salonen R: Increased risk of non-insulin dependent diabetes mellitus at low plasma vitamin E concentrations: a four year follow up study in men. BMJ 1995, 311:1124-1127.

53. American Dietetic Association; Dietitians of Canada: Position of the American dietetic association and dietitians of Canada: vegetarian diets. J Am Diet Assoc 2003, 103(6):748-765.

54. Chaussain JL, Georges P, Gendrel D, Donnadieu M, Job JC: Serum branched-chain amino acids in the diagnosis of hyperinsulinism in infancy. J Pediatr 1980, 97:923-926.

55. Van Dam RM, Willett WC, Rimm EB, Stampfer MJ, Hu FB: Dietary fat and meat intake in relation to risk of type 2 diabetes in men. Diabetes Care 2002, 25:417-424.

56. Aune D, Ursin G, Veierød MB: Meat consumption and the risk of type 2 diabetes: a systematic review and meta-analysis of cohort studies. Diabetologia 2009, 52(11):2277-2287.

57. InterAct Consortium: Association between dietary meat consumption and incident type 2 diabetes: the EPIC-InterAct study. Diabetologia 2013, 56(1):47-59.

58. Schulze MB, Manson JE, Willett WC, Hu FB: Processed meat intake and incidence of type 2 diabetes in younger and middle-aged women. Diabetologia 2003, 46:1465-1473.

59. Song Y, Manson JE, Buring JE, Liu S: A prospective study of red meat consumption and type 2 diabetes in middle-aged and elderly women: the women's health study. Diabetes Care 2004, 27:2108-2115.

60. Pan A, Sun Q, Bernstein AM, Manson JE, Willett WC, Hu FB: Changes in red meat consumption and subsequent risk of type 2 diabetes mellitus: three cohorts of US men and women. JAMA Intern Med 2013, 173(14):1328-1335.

61. Villegas R, Shu XO, Gao YT, Yang G, Cai H, Li H, Zheng W: The association of meat intake and the risk of type 2 diabetes may be modified by body weight. Int J Med Sci 2006, 3:152-159.

62. Simmons RK, Harding AH, Wareham NJ, Griffin SJ: Do simple questions about diet and physical activity help to identify those at risk of type 2 diabetes? Diabet Med 2007, 24:830-835

63. Lee $\mathrm{DH}$, Folsom $\mathrm{AR}$, Jacobs $\mathrm{DR}$ Jr: Dietary iron intake and type 2 diabetes incidence in postmenopausal women: the lowa women's health study. Diabetologia 2004, 47:185-194.

64. Schulze MB, Hoffmann K, Boeing H, Linseisen J, Rohrmann S, Möhlig M, Pfeiffer AF, Spranger J, Thamer C, Häring HU, Fritsche A, Joost HG: An accurate risk score based on anthropometric, dietary, and lifestyle factors to predict the development of type 2 diabetes. Diabetes Care 2007, 30:510-515.

65. Hodge AM, English DR, O'Dea K, Giles GG: Dietary patterns and diabetes incidence in the Melbourne collaborative cohort study. Am J Epidemiol 2007, 165:603-610.

66. Key T, Davey G: Prevalence of obesity is low in people who do not eat meat. Br Med J 1996, 313:816-817.

67. Misra A, Pandey RM, Devi JR, Sharma R, Vikram NK, Khanna N: High prevalence of diabetes, obesity and dyslipidaemia in urban slum population in northern India. Int J Obes Relat Metab Disord 2001, 25:1722-1729.

68. Ramachandran A, Snehalatha C, Vijay V: Low risk threshold for acquired diabetogenic factors in Asian Indians. Diabetes Res Clin Pract 2004, 65:189-195.

69. Mohan V, Deepa M, Deepa R, Shanthirani CS, Farooq S, Ganesan A, Datta M: Secular trends in the prevalence of diabetes and impaired glucose tolerance in urban South India -the Chennai Urban Rural Epidemiology Study (CURES-17). Diabetologia 2006, 49:1175-1178.

70. Tiwari RR, Deb PK, Debbarma A, Chaudhuri R, Chakraborti A, Lepcha M, Chakraborti G: Risk factor analysis in self-reported diabetes in a rural Kerala population. Int J Diabetes Dev Ctries 2008, 28:91-94. 
71. Vijayakumar G, Arun R, Kutty VR: High prevalence of type 2 diabetes mellitus and other metabolic disorders in rural Central Kerala. J Assoc Physicians India 2009, 57:563-567.

72. Corsi DJ, Subramanian SV: Association between socioeconomic status and self-reported diabetes in India: a cross-sectional multilevel analysis. BMJ Open 2012, 2:e000895. doi:10.1136/bmjopen-2012-000895, 2012

73. Ebrahim S, Kinra S, Bowen L, Andersen E, Ben-Shlomo Y, Lyngdoh T, Ramakrishnan L, Ahuja RC, Joshi P, Mohan Das S, Mohan M, Davey Smith G, Prabhakaran D, Reddy KS, for the Indian Migration Study group: The effect of rural-to-urban migration on obesity and diabetes in India: a cross-sectional study. PLoS Med 2010, 7(4):e1000268. doi:10.1371/journal.pmed.1000268.

74. Agrawal S, Ebrahim S: Prevalence and risk factors for self-reported diabetes among adult men and women in India: findings from a national cross-sectional survey. Publ Health Nutr 2011, 15:1065-1077.

75. Okura Y, Urban LH, Mahoney DW, Jacobsen SJ, Rodeheffer RJ: Agreement between self-report questionnaires and medical record data was substantial for diabetes, hypertension, myocardial infarction and stroke but not for heart failure. J Clin Epidemiol 2004, 57:1096 e103.

76. Subramanian SV, Subramanyam MA, Selvaraj S, Kawachi I: Are self-reports of health and morbidities in developing countries misleading? Evidence from India. Soc Sci Med 2009, 68:260 e5.

77. Registrar General of India: Census of India: C-13 Single Year Age Returns by Residence and Sex. 2001. http://www.censusindia.gov.in/Tables_Published/CSeries/c_series_tables_2001.aspx, 2001.

78. Mohan V, Mathur P, Deepa R, Deepa M, Shukla DK, Menon GR, Anand K, Desai NG, Joshi PP, Mahanta J, Thankappan KR, Shah B: Urban rural differences in prevalence of self-reported diabetes in India-the WHOICMR Indian NCD risk factor surveillance. Diabetes Res Clin Pract 2008, 80:159-168.

79. Pullum TW: An Assessment of the Quality of Data on Health and Nutrition in the DHS Surveys, 1993-2003. Methodological Reports No. 6. Calverton, MD: Macro International Inc; 2008.

80. Patel PS, Sharp SJ, Luben RN, Khaw KT, Bingham SA, Wareham NJ, Forouhi NG: Association between type of dietary fish and seafood intake and the risk of incident type 2 diabetes: the European Prospective Investigation of Cancer (EPIC)-Norfolk cohort study. Diabetes Care 2009, 32:1857-1863.

doi:10.1186/1475-2891-13-89

Cite this article as: Agrawal et al:: Type of vegetarian diet, obesity and diabetes in adult Indian population. Nutrition Journal 2014 13:89.

\section{Submit your next manuscript to BioMed Central and take full advantage of:}

- Convenient online submission

- Thorough peer review

- No space constraints or color figure charges

- Immediate publication on acceptance

- Inclusion in PubMed, CAS, Scopus and Google Scholar

- Research which is freely available for redistribution

Submit your manuscript at www.biomedcentral.com/submit
C Biomed Central 